\title{
Les objets connectés pour améliorer la culture de la production épurée : revue de littérature et esquisse de solution applicable aux entreprises
} manufacturières

\section{Industrial Internet of Things to improve lean manufacturing culture: literature review and solution outline for manufacturing plants}

\author{
Didérot Déraillet Tadja ${ }^{1,2}$, Samuel Bassetto ${ }^{3}$, Michel Tollenaere ${ }^{4}$, Tony Wong ${ }^{5}$ \\ ${ }^{1}$ Département de génie industriel, Polytechnique Montréal, Montréal, Canada, diderot-deraillet.tadja@polymtl.ca \\ ${ }^{2}$ Département de génie industriel, Collège Ahuntsic, Montréal, Canada, diderot-deraillet.tadja@collegeahuntsic.qc.ca \\ ${ }^{3}$ Département de génie industriel, Polytechnique Montréal, Montréal, Canada, samuel-jean.bassetto@polymtl.ca \\ ${ }^{4}$ Institut National Polytechnique de Grenoble - Génie industriel, Grenoble, France, michel.tollenaere@grenoble-inp.fr \\ ${ }^{5}$ Département de génie des systèmes, École de technologie supérieure, Montréal, Canada, tony.wong@etsmtl.ca
}

RÉSUMÉ. Ce travail vise à montrer l'importance des objets connectés pour améliorer la culture de la production épurée. Afin d'atteindre cet objectif, la revue de littérature qui englobe la production épurée, l'industrie 4.0 et le comportement des équipes de travail a été basée sur le processus de revue de littérature adapté de l'Université Concordia (2020) et de Machi (2012). À la fin de ce travail, il a été possible de structurer les pratiques techniques de la production épurée en 5 méthodes et d'identifier 8 principaux groupes technologiques de l'industrie 4.0. Le fruit qui ressort de ce travail est une proposition applicable aux entreprises manufacturières. Celle-ci est le système "lean coach CNV » basé sur les objets connectés dont l'application a le potentiel de promouvoir la culture de la production épurée. En plus de constituer une source d'actualisation de l'enseignement de la production épurée, sur le plan pratique, la proposition contenue dans ce travail est une piste de solution au problème connu de l'échec de mise en œuvre de la production épurée au sein des entreprises manufacturières.

ABSTRACT. This work aims to show importance of Industrial Internet of Things to improve lean manufacturing culture. The literature review that encompasses lean manufacturing, industry 4.0 and teamwork behaviour, is based on Concordia University Library (2020) and Machi (2012) literature review processes. From this work, it has been possible to structure lean manufacturing technical practices into 5 methods and identify 8 main industry 4.0 technological groups. The fruit of this work is a NVC lean coach system based on the integration of industrial internet of thing to seed lean manufacturing culture. Although this system is at proposal stage, it is a potential solution to the known problem of lean manufacturing implementation failure in manufacturing plants. At teaching level, it will contribute to update lean manufacturing courses.

MOTS-CLÉS. Production épurée, Objets connectés, Comportement des équipes de travail.

KEYWORDS. Lean manufacturing, Industrial Internet of things, Teamwork behaviour.

\section{Introduction}

Depuis les débuts de l'ère industrielle, l'un des défis majeurs des entreprises manufacturières a toujours été de mieux produire grâce à des méthodes qui ultimement permettent de faire plus avec moins. C'est le cas particulier des Petites et Moyennes Entreprises (PME) dont l'existence même dépend de leurs capacités à offrir aux clients situés dans les quatre coins du monde des produits de bonne qualité, au coût abordable, en quantité suffisante et surtout à l'endroit et à l'instant indiqué par ces clients. Lorsque ces défis sont relevés, il en découle une bonne santé de l'économie, l'amélioration du climat social et même la fierté des populations pour ne citer que ces exemples. Mais, force est de constater que la majorité des pays industrialisés connaissent une baisse remarquable de leur productivité ces dernières décennies (Jahchan, 2016). Tout particulièrement, le secteur manufacturier de la province du Québec au Canada accuse un retard sur le plan de la 
productivité par rapport à la moyenne canadienne et cet écart s'est significativement creusé entre 2014 et 2018 (STIQ, 2020). Pourtant, il était prévu que la grande diffusion des technologies numériques qui a commencé aux années 2000 devait déclencher une nouvelle vague de croissance de la productivité semblable à celle observée dans le passé, à partir du milieu des années 1880, en raison de l'électrification des moyens de production (OCDE, 2021).

Le contraste entre la prolifération des technologies numériques et la décroissance des gains de productivité a amené certains auteurs à raviver le paradoxe de la productivité qui avait été évoqué par l'économiste Robert Solow en disant «on voit des ordinateurs partout, sauf dans les données sur la productivité »(Solow, 1987). Quoi que l'on dise, la faible productivité d'une entreprise manufacturière reflète indiscutablement la présence des gaspillages multiformes en son sein. Les comportements et les actions favorables à la production épurée ou plus généralement l'opérationnalisation de la production épurée seraient une solution à cette situation. En effet, l'implémentation réussie de la production épurée dans une entreprise manufacturière en Inde a récemment abouti à des économies de 33 heures de travail par semaine (Karikalan et al., 2019). Celles-ci étant convertibles en accroissement de la productivité.

Malgré la pertinence incontestée de la production épurée pour l'amélioration de la productivité, plusieurs entreprises se battent encore pour parvenir à l'opérationnaliser et échouent souvent à atteindre les résultats escomptés (Pearce et al., 2018). Cette production épurée qui a été impulsée depuis 1954 par Ohno et Shingo (Zajkowska, 2013), est un système centré sur les personnes où celles-ci sont directement impliquées dans les processus d'amélioration continue et doivent être actives dans la résolution des problèmes (Liker, 2004). Or il se trouve qu'au cours de l'année 2020 en Amérique du Nord (Canada et États-Unis), 66\% des employés étaient désengagés ou activement désengagés (Gallup, 2021). Dans les autres pays industrialisés, la situation est loin d'être meilleure. Bien évidemment, si dans une équipe de travail il y a du désengagement et les comportements des membres ne sont pas favorables, celle-ci ne peut pas prospérer et les bonnes pratiques opérationnelles ne peuvent pas être appliquées. C'est d'ailleurs ce que mentionne l'étude de Secchi (2019), qui de façon résumée indique que les comportements humains et organisationnels sont les principales causes des échecs de mise en œuvre de la production épurée (Secchi \& Camuffo, 2019). Sans que celle-ci soit l'unique cause, la prise en compte du comportement des équipes de travail semble donc échapper aux entreprises manufacturières qui se lancent dans les projets d'implémentation de la production épurée. Il existe un réel problème de culture de la production épurée au sein des entreprises manufacturières.

Les gains de productivité attendus avec l'arrivée des technologies numériques pourraient peutêtre s'obtenir en mettant ces technologies à contribution pour réinventer les méthodes de production et surtout de promouvoir une culture de la production épurée au sein des équipes de travail. La littérature scientifique à ce sujet propose des possibilités d'exploitation des technologies de l'industrie 4.0 pour numériser les outils techniques de la production épurée. Toutefois, la quasitotalité de ces propositions n'élucide pas l'exploitation de ces technologies pour la promotion d'une culture de la production épurée. Il devient donc possible de se poser la question suivante : dans un système manufacturier, est-ce qu'une approche basée sur l'intégration des objets connectés peut contribuer à l'adoption des comportements favorables à la production épurée ?

Cet article commence par une synthèse de la littérature centrée sur la production épurée, le changement de comportement au sein des équipes de travail et de l'application des technologies de l'industrie 4.0 à cette fin. Ensuite, un système d'accompagnement le «lean coach $C N V$ » qui couple la communication non violente à un outil technique dédié à la détection automatique des 7 gaspillages est proposée afin de montrer une application possible des objets connectés pour la promotion des comportements favorables à la production épurée au sein des équipes de travail en milieu manufacturier. 


\section{Méthodologie}

La stratégie qui a guidé la rédaction de ce papier consiste à fouiller la littérature pour donner une réponse aux questions suivantes :

1) Qu'est-ce que la production épurée et quelles sont les pratiques de la production épurée ?

2) Qu'est-ce que l'industrie 4.0 et comment se conjugue-t-elle avec la production épurée ?

3) Quels sont les leviers d'actions pour amener une équipe de travail à adopter une nouvelle culture de travail ?

Le processus recommandé par la librairie de l'Université Concordia (2020) a été appliqué pour mener cette revue de littérature (Concordia University, 2020). Comme le montre la figure 2.1, il s'agit d'un processus en 4 étapes. Il est comparable à celui de Machi (Machi \& McEvoy, 2012), mais a l'avantage d'être pragmatique et donc facile à appliquer. En effet, il ne contraint pas l'auteur de suivre des tâches spécifiques à chaque tape. Cependant, le processus de Machi (2012) qui est très détaillé a servi de complément à ce processus notamment pour organiser et gérer les informations collectées.

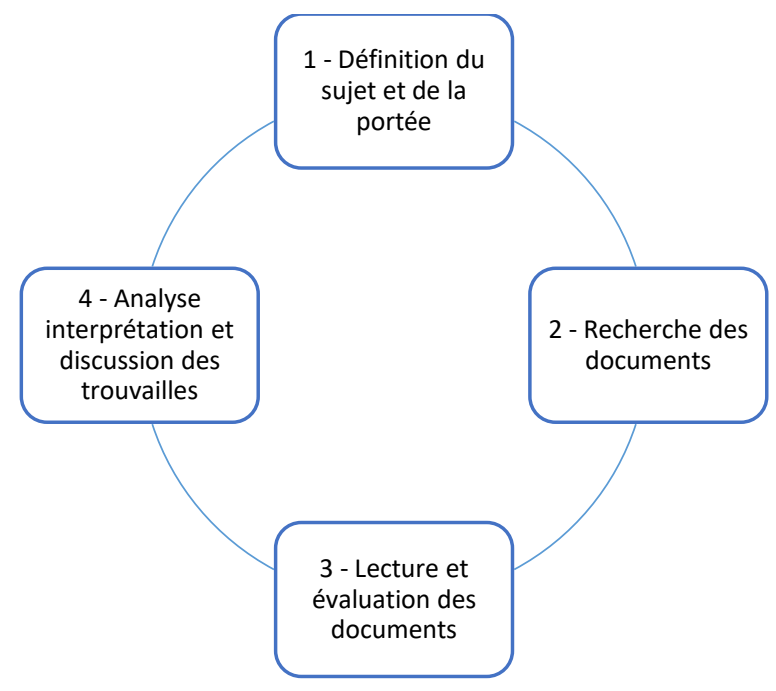

Figure 2.1. Processus de revue de littérature. Adapté de Concordia University (2020)

Bien que toutes les étapes de ce processus soient indispensables, nous pensons que l'étape de recherche des documents a été l'étape critique. En effet, le contenu de cette revue de littérature dépend directement des documents qui ont été trouvés et analysés. La recherche des documents en général et des journaux scientifiques en particulier s'est appuyée sur un plan de 4 concepts à savoir la production épurée, l'industrie 4.0, le changement comportemental et les systèmes manufacturiers.

Les bases de données que sont Compendex, Web of Science et Apa Psycnet ont été interrogées sur une période allant de 2010 à 2020 par combinaisons de ces 4 concepts. D'autres bases de données comme Researchgate et Googlescholar ont aussi été consultées pour trouver des documents complémentaires. Le tableau 2.1 résume la combinaison des mots-clés utilisés pour interroger les bases de données et trouver les références pour traiter les questions que cette revue permet de répondre. 


\begin{tabular}{|c|c|}
\hline Question traitée & Requête.s utilisée.s \\
\hline \multirow{2}{*}{$\begin{array}{l}\text { Qu'est-ce que la production } \\
\text { épurée et quelles sont les } \\
\text { pratiques de la production } \\
\text { épurée? }\end{array}$} & $\begin{array}{l}\text { (Lean manufacturing OR Lean production OR Toyota production system OR Continuous } \\
\text { improvement OR Muda) }\end{array}$ \\
\hline & $\begin{array}{l}\text { (Lean manufacturing OR Lean production OR Toyota production system OR Continuous } \\
\text { improvement OR Muda) AND (Manufacturing plant OR Factory OR Production system OR } \\
\text { Industry OR Assembly line) }\end{array}$ \\
\hline \multirow{2}{*}{$\begin{array}{l}\text { Qu'est-ce l'industrie } 4.0 \text { et } \\
\text { comment se conjugue-t-elle } \\
\text { avec la production épurée? }\end{array}$} & $\begin{array}{l}\text { (Internet of Things OR IoT OR IIoT OR IoD OR Industry 4.0 OR Connected objects OR Lean } \\
\text { 4.0 OR Smart manufacturing) }\end{array}$ \\
\hline & $\begin{array}{l}\text { (Internet of Things OR IoT OR IIoT OR IoD OR Industry 4.0 OR Connected objects OR Lean } \\
\text { 4.0 OR Smart manufacturing) AND (Lean manufacturing OR Lean production OR Toyota } \\
\text { production system OR Continuous improvement OR Muda) }\end{array}$ \\
\hline \multirow{4}{*}{$\begin{array}{l}\text { Quels sont les leviers } \\
\text { d'actions pour amener une } \\
\text { équipe de travail à adopter } \\
\text { une nouvelle pratique de } \\
\text { travail? }\end{array}$} & $\begin{array}{l}\text { (Commitment OR Engagement OR Virtuou* OR Virtuous Behavio* OR Persuasion OR } \\
\text { Persuasive technolog* OR Captolog* OR Behavio* chang* OR Attitud* chang* OR Habit* } \\
\text { chang* OR Lean Behavio*) }\end{array}$ \\
\hline & $\begin{array}{l}\text { (Commitment OR Engagement OR Virtuou* OR Virtuous Behavio* OR Persuasion OR } \\
\text { Persuasive technolog* OR Captolog* OR Behavio* chang* OR Attitud* chang* OR Habit* } \\
\text { chang** OR Lean Behavio*) AND (Lean manufacturing OR Lean production OR Toyota } \\
\text { production system OR Continuous improvement OR Muda) }\end{array}$ \\
\hline & $\begin{array}{l}\text { (Commitment OR Engagement OR Virtuou* OR Virtuous Behavio* OR Persuasion OR } \\
\text { Persuasive technolog* OR Captolog* OR Behavio* chang* OR Attitud* chang* OR Habit* } \\
\text { chang** OR Lean Behavio*) AND (Internet of Things OR IoT OR IIoT OR IoD OR Industry } \\
\text { 4.0 OR Connected objects OR Lean 4.0 OR Smart manufacturing) }\end{array}$ \\
\hline & $\begin{array}{l}\text { (Commitment OR Engagement OR Virtuou* OR Virtuous Behavio* OR Persuasion OR } \\
\text { Persuasive technolog* OR Captolog* OR Behavio* chang* OR Attitud* chang* OR Habit* } \\
\text { chang* OR Lean Behavio*) AND (Internet of Things OR IoT OR IIoT OR IoD OR Industry } \\
\text { 4.0 OR Connected objects OR Lean } 4.0 \text { OR Smart manufacturing) AND (Lean manufacturing } \\
\text { OR Lean production OR Toyota production system OR Continuous improvement OR Muda) }\end{array}$ \\
\hline
\end{tabular}

Tableau 2.1. Requêtes formulées pour l'interrogation des bases de données

De tous les documents qui ont été trouvés, le résumé, les mots clés, la date de parution et le type de documents ont été utilisés comme critères de sélection des documents à utiliser pour la revue de littérature que nous présentons à partir de la section suivante.

\section{Revue de littérature}

\subsection{Qu'est-ce que la production épurée?}

Le lean manufacturing est l'expression anglaise utilisée pour désigner la production épurée. En anglais, «lean » signifie maigre. Dans les entreprises «maigres », « dégraissées » et économiques, le «gras » renvoi aux opérations qui n'apportent pas de valeur ajoutée du point de vue du client (Zajkowska, 2013). Cette traduction littérale du lean manufacturing ou production amincie ou alors production épurée nous laisse voir que le lean manufacturing est fondamentalement orienté vers l'amélioration de la santé des entreprises manufacturières. La production épurée est un ensemble de principes et techniques de gestion visant à éliminer les gaspillages dans un système manufacturier et à augmenter le flux d'activités qui du point de vue des clients, ajoutent de la valeur au produit (Womack \& Jones, 2003). Un système manufacturier dont la gestion est basée sur ces principes et techniques fonctionnera en étant débarrassé des gaspillages qui minent les dimensions de sa performance opérationnelle en plus d'être enrichis d'activités qui créent de la valeur aux clients. Dans de telles conditions, les clients sont satisfaits car il en découle un meilleur rapport qualité prix. L'entreprise est aussi satisfaite, car les coûts de production sont réduits pour une meilleure marge de 
profit. Il s'agit donc d'une forme de gestion optimale où les deux principales parties prenantes (fournisseurs et clients) sont toutes gagnantes.

Lorsqu'un client évalue un produit ou un service, il évalue sa valeur perçue par rapport au prix demandé (Almquist et al., 2016). Donc du point de vue du client, la valeur ajoutée n'est qu'une perception qui crée un sentiment de satisfaction et le convainc à payer le prix d'acquisition du bien. Pour une entreprise, la valeur ajoutée est le fruit des opérations de transformation d'un bien ou d'un service à partir d'une valeur initiale acquise sur le marché des valeurs. D'après l'Institut National de la Statistique et des Études Économiques (INSEE), elle est égale à la valeur de la production diminuée de la consommation intermédiaire (INSEE, 2020). La création ou l'augmentation de la valeur provient donc essentiellement de la diminution des consommations intermédiaires auxquelles sont rattachées les opérations sans valeur ajoutée. Cette création de valeur dans les entreprises manufacturières correspond à ce qui convient de désigner par production à valeur ajoutée. Selon le ministère de l'économie et de l'innovation du Québec, la production à valeur ajoutée permet à l'organisation de se doter d'une grande souplesse lui permettant de produire sur commande au prix de la production de masse, en petite quantité ou à l'unité en s'ajustant rapidement aux goûts changeant des consommateurs (Gouvernement du Québec, 2020). La production épurée, est alors un prolongement de la production à valeur ajoutée. En effet, elle permet à l'entreprise de créer de la valeur à la fois pour elle et ses clients.

\subsection{Qu'est-ce que les 7 gaspillages ?}

La principale cause de non-valeur ajoutée dans un système de production de biens est le gaspillage. Un gaspillage est tout sauf la quantité minimale d'équipements, matériaux, composants et temps de travail qui sont absolument essentiels à la production (Taj \& Morosan, 2011). Ohno (1988) et Shingo (1989) qui sont les précurseurs de la production épurée ont identifié 7 types de gaspillage qui peuvent se manifester dans un système de production. Il s'agit de la surproduction, les temps d'attente, les transports inutiles, les opérations de traitement excessif, les stocks inutiles, les mouvements non nécessaires, la non-qualité. Le tableau 3.1 (Thürer et al., 2017) qui suit résume une description de chaque type de gaspillage tels que formulé par (Ohno, 1988), (Shingo, 1989) et (Liker, 2004).

\begin{tabular}{|c|c|c|c|}
\hline $\begin{array}{l}\text { Type de } \\
\text { gaspillage }\end{array}$ & Ohno (1988) & Shingo (1989) & Liker (2004) \\
\hline Overproduction & $\begin{array}{l}\text { Process transformation } \\
\text { without need e.g. to } \\
\text { avoid waiting. }\end{array}$ & $\begin{array}{l}\text { Part of operation. Anticipated } \\
\text { process transformations; } \\
\text { producing too much. }\end{array}$ & $\begin{array}{l}\text { Producing items for which there } \\
\text { are no orders, which generates } \\
\text { such wastes as overstaffing and } \\
\text { storage and transportation costs } \\
\text { because of excess inventory. }\end{array}$ \\
\hline $\begin{array}{l}\text { Waiting (time on } \\
\text { hand) }\end{array}$ & $\begin{array}{l}\text { Waste of time at hand. } \\
\text { Any delay in the actions } \\
\text { that accomplish process } \\
\text { transformation }\end{array}$ & $\begin{array}{l}\text { Part of operations. Any delay in } \\
\text { the actions that accomplish } \\
\text { process transformations. }\end{array}$ & $\begin{array}{l}\text { Workers merely serving to watch } \\
\text { an automated machine or having to } \\
\text { stand around waiting for the next } \\
\text { processing step, tool, supply, part, } \\
\text { etc., or just plain having no work } \\
\text { because of stock-outs, lot } \\
\text { processing delays, equipment } \\
\text { downtime, and capacity } \\
\text { bottlenecks. }\end{array}$ \\
\hline $\begin{array}{c}\text { Type de } \\
\text { gaspillage (suite) }\end{array}$ & Ohno (1988) & Shingo (1989) & Liker (2004) \\
\hline
\end{tabular}




\begin{tabular}{|c|c|c|c|}
\hline $\begin{array}{l}\text { Unnecessary } \\
\text { transport or } \\
\text { conveyance }\end{array}$ & $\begin{array}{l}\text { Waste of carrying. Any } \\
\text { movement of material or } \\
\text { products. }\end{array}$ & $\begin{array}{l}\text { Part of process. Any movement } \\
\text { of material or products. }\end{array}$ & $\begin{array}{l}\text { Carrying work in process (WIP) } \\
\text { long distances, creating inefficient } \\
\text { transport, or moving materials, } \\
\text { parts, or finished goods into or out } \\
\text { of storage or between processes }\end{array}$ \\
\hline $\begin{array}{l}\text { Overprocessing } \\
\text { or incorrect } \\
\text { processing }\end{array}$ & $\begin{array}{l}\text { Waste of processing } \\
\text { itself. Taking unneeded } \\
\text { steps to process the parts. }\end{array}$ & $\begin{array}{l}\text { Part of process. Producing } \\
\text { anything which is not valued by } \\
\text { the customer. Value engineering } \\
\text { and value analysis must be } \\
\text { carried out first. Instead of } \\
\text { focusing merely on efficiency, } \\
\text { the question is why we make a } \\
\text { given product and use a given } \\
\text { method. }\end{array}$ & $\begin{array}{l}\text { Taking unneeded steps to process } \\
\text { the parts. Inefficiently processing } \\
\text { due to poor tool and product } \\
\text { design, causing unnecessary } \\
\text { motion and producing defects. } \\
\text { Waste is generated when providing } \\
\text { higher-quality products than is } \\
\text { necessary. }\end{array}$ \\
\hline Excess inventory & $\begin{array}{l}\text { Waste of stock at hand. } \\
\text { Work-in-Process and } \\
\text { Finished Goods } \\
\text { Inventory }\end{array}$ & $\begin{array}{l}\text { Part of process. Work-in } \\
\text { Process (and related process } \\
\text { delays) and Finished Goods } \\
\text { Inventory }\end{array}$ & $\begin{array}{l}\text { Excess raw material, WIP, or } \\
\text { finished goods causing longer lead } \\
\text { times, obsolescence, damaged } \\
\text { goods, transportation and storage } \\
\text { costs, and delay }\end{array}$ \\
\hline $\begin{array}{l}\text { Unnecessary } \\
\text { movement }\end{array}$ & $\begin{array}{l}\text { Waste of movement. } \\
\text { Any motion which does } \\
\text { not transform the product } \\
\text { adding value. }\end{array}$ & $\begin{array}{l}\text { Part of operations. Any action } \\
\text { which does not transform the } \\
\text { product adding value. }\end{array}$ & $\begin{array}{l}\text { Any wasted motion employees } \\
\text { have to perform during the course } \\
\text { of their work, such as looking for, } \\
\text { reaching for, or stacking parts, } \\
\text { tools, etc. }\end{array}$ \\
\hline Defects & $\begin{array}{l}\text { Waste of making } \\
\text { defective products. }\end{array}$ & $\begin{array}{l}\text { Part of process. Any quality } \\
\text { loss, expressed as rework or } \\
\text { scrap. }\end{array}$ & $\begin{array}{l}\text { Production of defective parts or } \\
\text { correction. Repair or rework, } \\
\text { scrap, replacement production, and } \\
\text { inspection mean wasteful } \\
\text { handling, time, and effort. }\end{array}$ \\
\hline
\end{tabular}

Tableau 3.1. Description pragmatique des 7 types de gaspillage

$\mathrm{Au}$ regard de ces descriptions, on peut réaffirmer qu'un gaspillage est une activité sans valeur ajoutée et lorsque ce type d'activités existent dans un système de production, elles augmentent en fin de compte le coût unitaire du produit. Ce coût devra être payé soit par les clients, soit par les fournisseurs, soit par l'employeur, les employés si ce n'est les gouvernements. Or ni l'une ni l'autre de ces parties ne sera prête à payer pour un gaspillage. La production épurée s'applique à toutes les activités de l'entreprise avec pour objectifs d'éliminer sinon réduire tous les gaspillages présents en son sein. Un système de gestion épuré permet aux entreprises de produire la quantité requise de produits ou de services de qualité, au bon moment, à la bonne place et au moindre coût (Stevenson \& Benedetti, 2011). Il s'agit des objectifs généraux de tout système de production. Lorsque ces objectifs sont atteints, ils lui confèrent au minimum une performance opérationnelle, financière et environnementale.

\subsection{Techniques de mise en œuvre de la production épurée}

\subsubsection{Identification des principaux outils techniques}

La pratique de la production épurée en milieu manufacturier se fait avec des outils techniques bien spécifiques. Dans la littérature, on retrouve une grande variété d'outils applicables à la production épurée. D'après Pavnaskar (2003), il en existe plus d'une centaine (Pavnaskar et al., 2003) et dans la vie d'une entreprise celle-ci pourra en plus développer des outils spécifiques à sa propre réalité. La production épurée est un système intégré de principes, pratiques, d'outils et de 
techniques (Čiarnienè \& Vienažindienė, 2015). Tous ces outils doivent travailler en synergie afin de tirer profit du système de gestion épurée. Bien qu'il existe une pléthore d'outils applicables à la production épurée, certains sont régulièrement présentés dans la littérature scientifique comme étant des incontournables pour les entreprises désireuses d'implémenter un système de gestion épurée. Les outils qui ont un caractère quantitatif comme le Value Stream Mapping (VSM), Single-Minute Exchange of Dies (SMED), le Kanban, l'Overall Efficiency Effectivness (OEE), bénéficient d'une plus grande attention parce qu'ils offrent la possibilité de prouver leurs effets sur les systèmes de production par des données chiffrées (Amrani \& Ducq, 2020). Dans l'industrie textile américaine, Hodge (2011) a identifié certains outils de gestion visuelle (le 5S et le VSM) comme étant ceux qui sont fréquemment utilisés par cette industrie (Hodge et al., 2011). Au sein des PME, Qing (2015) a identifié après une revue de littérature systématique que le 5S, le Kanban, le travail standard, et le VSM sont les outils les plus utilisés par les PME pour opérationnaliser la production épurée (Hu Qing et al., 2015).

Le secteur d'activité et la taille de l'entreprise sont les facteurs déterminants pour le choix du type d'outils à utiliser au déploiement de la production épurée dans une entreprise. Les PME ont tendance à être plus sélectives dans le choix de ces outils que les grandes entreprises à cause des contraintes techniques, financières et de temps (Mathur et al., 2012). Même si le secteur d'activité et la taille de l'entreprise influencent le choix des outils techniques de la production épurée, certains comme le montre la figure 3.1 sont régulièrement cités et reconnus comme étant les plus classiques pour la mise en œuvre de la production épurée. Cette figure est un classement de Pareto des pratiques de la production épurée dans l'industrie italienne (Bevilacqua et al., 2017).

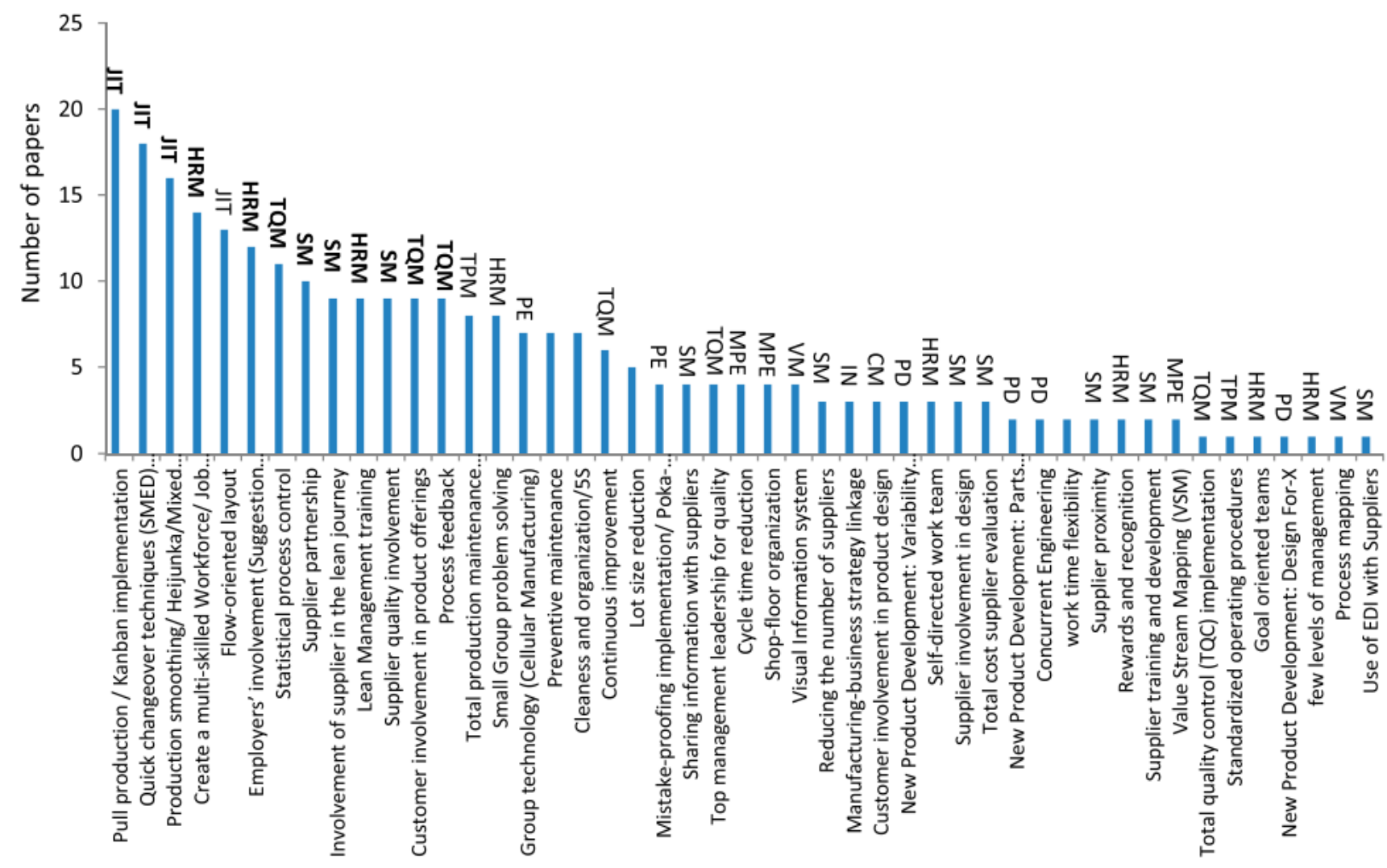

Figure 3.1. Outils techniques de la production épurée. Adapté de (Bevilacqua et al., 2017)

\subsubsection{Identification des principales méthodes}

Après avoir identifié les outils techniques classiques de la production épurée à la section précédente, ceux-ci peuvent être regroupés selon les objectifs visés de leurs utilisations pour structurer un modèle de gestion basé sur la production épurée. Il existe plusieurs façons de regrouper les outils techniques afin de représenter la nature multidimensionnelle de la production épurée (Shah 
\& Ward, 2003). Parmi elles, le degré d'adoption dans la littérature et l'analyse factorielle (Negrão et al., 2017).

En utilisant l'analyse factorielle, Shah (2003) a formé 4 groupes méthodologiques que sont le Juste-à-Temps (JIT), la Total Productive Maintenance (TPM), la Total Quality Management (TQM) et le Human Resource Management (HRM) indispensables à la mise en œuvre d'un système de gestion de la production épurée (Shah \& Ward, 2003). Dans son travail de thèse, Lyonnet (2010) a identifié par consensus entre les pratiques au sein des entreprises et les publications scientifiques que Waste Hunting (WH), le JIT, la Qualité, le Continuous Improvement (CI), le Visual Management (VM) et le HRM sont les 6 regroupements possibles de l'ensemble des outils de la production épurée (Lyonnet, 2010). Belekoukias (2014) quant à lui, a montré que le JIT, la TPM, le Value Stream Mapping (VSM), le CI et l'Autonomisation sont les 5 principales méthodes de la production épurée auxquelles peuvent se rattacher l'ensemble de ses outils techniques (Belekoukias et al., 2014).

Sur la base des travaux de ces chercheurs, nous pouvons observer que dans la littérature, il n'y a pas unanimité sur les méthodes qui constituent un système de la production épurée. Le tableau 3.2 qui suit résume l'ensemble des méthodes de la production épurée identifiée par ces chercheurs qui en réalité est le fruit des synthèses de plusieurs travaux précédents leurs publications.

\begin{tabular}{|l|c|c|c|c|c|c|c|c|}
\hline & JIT & TPM & TQM & CI & HRM & VM & WH & VSM \\
\hline Shah (2003) & 1 & 1 & 1 & 0 & 1 & 0 & 0 & 0 \\
\hline Lyonnet (2010) & 1 & 0 & 1 & 1 & 1 & 1 & 1 & 0 \\
\hline Belekoukias (2014) & 1 & 1 & 1 & 1 & 0 & 0 & 0 & 1 \\
\hline
\end{tabular}

1= La méthode fait partie du système de la production épurée selon l'auteur

Tableau 3.2. Synthèse des méthodes de la production épurée

À partir du tableau 3.2, on peut déduire le tableau 3.3 qui donne la fréquence empirique de chaque méthode obtenue à l'aide de la formule [1].

$$
E(x) \approx \frac{1}{N} \sum_{i=1}^{N} x_{i}=p(x)
$$

\begin{tabular}{|l|c|c|c|c|c|c|c|c|}
\hline & JIT & TPM & TQM & CI & HRM & VM & WH & VSM \\
\hline Fréquence (\%) & 100 & 66.66 & 100 & 66.66 & 66.66 & 33.33 & 33.33 & 33.33 \\
\hline
\end{tabular}

Tableau 3.3. Fréquence empirique des méthodes de la production épurée

Ce tableau nous permet de constater que le JIT, la TPM, la TQM, la CI et le HRM sont plus fréquemment identifiées dans Shah (2003), Lyonnet (2010) et Belekoukias (2014) comme étant les méthodes d'un système de la production épurée. Nous décrivons de façon succincte chacune de celles-ci dans les sections qui suivent et les retenons dans cet article pour désigner les principales méthodes d'un système de gestion de la production épurée.

\subsubsection{Le Juste-à-Temps}

Le Juste-à-Temps (JIT) a été introduit pour la première fois au Japon vers les années 1970 (Yash, 2016). Aussi appelé Heijunka en japonais, il signifie lissage de la production en volume et mix de produit (Lyonnet et al., 2010). C'est une méthode de planification et de contrôle de la production 
pilotée par la commande client qui marque le déclenchement des opérations. Une méthode de production basée sur le JIT permet aux entreprises de fonctionner en flux tirés.

Il a été montré empiriquement que le JIT a un effet positif sur la performance environnementale des entreprises (Garza-Reyes et al., 2018). En produisant les justes produits en justes quantités et au juste moment, il en découle inévitablement des économies en gestion des stocks qui sont souvent des opérations coûteuses et énergivores. Par contre les ruptures d'approvisionnement, les pannes des machines, l'absentéisme sont des aléas qui minent l'objectif premier du JIT (Lyonnet et al., 2010). Pour ces raisons, l'opérationnalisation de cette méthode requiert une très bonne flexibilité, agilité et fiabilité du système de production. En plus, il faut une bonne maîtrise des temps opérationnels, une configuration simplifiée du système de production et des relations-fournisseurs basées sur un partenariat. Le JIT peut être associé à la fonction production d'une entreprise manufacturière comme méthode de travail. Le Single Minute Exchange of Dies (SMED), le Kanban, l'Overall Equipment Effectivness (OEE) sont quelques outils techniques rattachés au JIT.

\subsubsection{La Total Productive Maintenance}

La Total Productive Maintenance (TPM) est un concept se définissant comme une maintenance productive qui implique les employés opérationnels de tous les niveaux hiérarchiques (Cua et al., 2001). À l'origine elle a été introduite sous la forme d'outils pratiques axés sur l'amélioration de la performance des équipements; elle est devenue aujourd'hui une approche intégrale centrée sur les équipements pour optimiser la productivité manufacturière (Ahuja \& Kumar, 2009). En plus, elle contribue à l'optimisation de la maintenance prédictive, préventive et corrective (Konecny \& Thun, 2011).

En TPM, opérateurs et personnels de maintenance travaillent en collaboration parfaite pour promouvoir une exploitation semi-autonome et totale des équipements de production par les opérateurs. Ainsi, les aléas de fonctionnement, les pannes de premier niveau et l'entretien de base de ces équipements sont directement résolus par les opérateurs. Comme impacts directs, les délais d'attente et les coûts de maintenance sont réduits. C'est pourquoi la TPM est un appui au JIT qui requiert une flexibilité et une agilité des moyens de production.

L'OEE est un outil technique qui permet de visualiser la pertinence et le niveau de succès de la TPM dans une entreprise. Plus la TPM est réussie dans une entreprise, meilleur sera son OEE. Mais, comme la TPM se fait avec les employés opérationnels de tous les niveaux, cela donne naissance à un défi de collaboration qui doit être surmonté pour prévenir les tensions et les conflits d'intérêts dans les équipes. Le personnel de maintenance doit former et céder une partie de ses responsabilités aux opérateurs qui n'ont pas nécessairement une technicité ce qui requiert de la patience. Donc, la mise en place de la TPM dans les entreprises doit nécessairement prendre en compte la gestion de la dynamique comportementale des équipes de travail.

\subsubsection{La Total Quality Management}

La Total Quality Management (TQM) est un ensemble de techniques et procédures utilisées pour réduire ou éliminer les variations observées dans les procédés de production ou dans les systèmes de service client avec pour but d'améliorer l'efficacité, la fiabilité et la qualité (Singh \& Singh, 2014). C'est aussi une philosophie de gestion holistique qui vise l'amélioration continue de toutes les fonctions d'une organisation (Kaynak, 2003).

L'objectif ultime de la TQM est la satisfaction et la fidélisation des clients internes et externes ce qui a un effet positif sur la pérennité de l'organisation. Au-delà des clients, la TQM vise également à satisfaire toutes les parties prenantes de la chaîne logistique: fournisseurs, collectivités et actionnaires (Lyonnet et al., 2010). Selon le modèle de la TQM, la qualité des produits et des procédés de production est la responsabilité de chacun et de tous impliqués dans la création ou la consommation des produits ou service (Dubey \& Singh, 2015). L'engagement des employés, la 
gestion par objectifs spécifiques mesurables et atteignables dans les délais sont les éléments d'une culture organisationnelle qui peuvent contribuer au succès de la TQM.

Il existe une complémentarité entre la TQM et le référentiel ISO 9000; un leadership positif, une approche par processus et une gestion des relations sont quelques principes de gestion identifiés par le référentiel ISO 9000 qui complètent le modèle de gestion de la TQM (Magd Hesham \& Curry Adrienne, 2003). Le Poka-Yoke, le contrôle statistique des procédés, le diagramme d'Ishikawa et les systèmes visuels de contrôle sont quelques outils techniques qui se rattachent à la TQM.

\subsubsection{Le Continuous Improvement}

Littéralement, Kaizen est un mot japonais composé de «Kai »et «Zen» qui signifient respectivement " changement » et " meilleur »; en anglais, cela correspond à " continuous improvement » et renvoi à tout effort systématique ayant pour but de trouver et appliquer les nouvelles façons de mieux faire un travail (Anand et al., 2009). En français, " continuous improvement » se traduit par «amélioration continue » soit, l'amélioration progressive, une démarche graduelle et douce de changement par opposition aux réformes brutales pour le meilleur (Lyonnet, 2010). Il s'agit d'un changement qui se fait en continu à la grandeur de l'entreprise. Comme la TPM et la TQM, le Continuous Improvement (CI) concerne l'ensemble du personnel à tous les niveaux hiérarchiques afin que les lendemains de l'entreprise soient meilleurs.

Le CI est avant tout une approche basée sur les processus et suppose que l'amélioration des performances peut être atteinte si et seulement si de meilleurs processus sont créés (Darlington et al., 2015). D'après Rossini (2019), un projet ou évènement Kaizen s'effectue en quatre phases à savoir la planification qui consiste à définir un planning des besoins en amélioration, le travail préparatoire qui est un travail d'analyse des besoins, l'identification des actions pour la solution et enfin la mise en œuvre des actions qui est l'évènement Kaizen proprement dit (Rossini, Audino, et al., 2019). La durée maximale d'un projet Kaizen est de 5 jours avec une équipe entièrement dédiée au projet (Rossini, Audino, et al., 2019). Lyonnet (2010) quant à elle, suggère que le processus d'un CI soit modélisé par la roue de Deming ou approche PDCA : Planifier (Plan), Réaliser (Do), Vérifier (Check) Agir (Act) (Lyonnet, 2010). La phase Plan consiste à spécifier les objectifs, les échéanciers et les moyens pour atteindre les résultats recherchés. La phase Do consiste à réaliser ce qui a été préalablement planifié, la phase Check ou phase de vérification est celle qui permet de s'assurer que les objectifs initialement définis sont atteints. Si les résultats ne sont pas atteints, la phase Act permet d'agir pour corriger ou ajuster les solutions. Le cycle recommence ainsi avec la phase Plan pour un nouveau besoin d'amélioration.

Le Kaizen ou CI est une méthode qui a la particularité d'être de courte durée pour des résultats concrets, visibles aux moindres coûts. À titre d'exemple, l'application du Kaizen a permis à une entreprise manufacturière dans le Wisconsin aux USA, d'améliorer en 2015 les délais de livraison de $17,8 \%$ et une réduction du temps d'attente de $91,13 \%$ entre les postes de travail (Lima et al., 2018). Puisque le Kaizen se fait aussi avec la participation de tous dans l'entreprise, cela contribue à favoriser une synergie d'équipe. Toutefois, comme le Kaizen repose sur de petits progrès, son impact peut être substantiellement faible avec le risque d'implanter les solutions non pérennes (Glover et al., 2015). Le Value Stream Mapping (VSM) est l'un des principaux outils techniques du Kaizen.

\subsubsection{Le Human Ressource Management}

La production épurée est une philosophie de gestion basée sur l'amélioration continue qui requiert l'implication et l'engagement de tous dans l'organisation (Womack \& Jones, 2003). En conséquence, la ressource humaine est un élément critique pour le déploiement de la production épurée. Tout le monde doit être impliqué et engagé. Dans une organisation qu'elle qu'est soit, si les personnes ne s'impliquent pas, ne se sentent pas concernées, ne collaborent pas, ne se respectent pas, sont violentes dans le langage, etc., pratiquement rien ne se fera. La gestion des ressources 
humaines en tant méthode de la production épurée inclus de façon non exhaustive les directives, les politiques, les programmes, les systèmes et les pratiques liées aux personnes qu'une organisation doit implémenter afin de gérer et améliorer les ressources humaines (Armstrong \& Taylor, 2014).

Lorsqu'une organisation adopte la production épurée comme mode de gestion, il s'agit d'un changement de paradigme qui commence par une gestion appropriée des ressources humaines (Yash, 2016). Le Human Resource Management (HRM) doit exister aussitôt qu'une relation de quelque forme que ce soit est établie entre l'employeur et l'employé (Cohen, 2015). Comme la ressource humaine est le dénominateur commun à toutes les méthodes de la production épurée, une action en faveur de celle-ci aura un effet positif sur la synergie entre les méthodes de la production épurée. Le principe de base du HRM est la responsabilisation et la motivation des employés (Furlan et al., 2011). C'est ce que confirment les pratiques HRM chez Toyota par exemple où les employés sont responsables de définir et améliorer leurs propres travaux (Spear \& Bowen, 1999). Aussi, la bonne gestion des ressources humaines se concrétise par des pratiques visant l'engagement des employés, la composition des équipes multifonctionnelles et la formation des employés.

\subsection{L'industrie 4.0 et la production épurée}

La production épurée était complètement indépendante de toute forme de technologies d'information et de communication dans sa forme originelle (Buer et al., 2018). Elle date des années 50 et ce n'est qu'en 1990 que l'idée d'établir un lien entre la production épurée et les technologies numériques a été impulsée avec le concept de "lean automation" (Womack et al., 1990). L'industrie 4.0 qui appartient à la grande famille des technologies numériques partage curieusement avec la production épurée le même objectif ultime qui est d'optimiser sinon améliorer au moins la dimension opérationnelle de la performance des entreprises. Dans cette section, nous identifions les principaux groupes technologiques de l'industrie 4.0 ensuite, nous explorons la synergie qui existe entre ces deux concepts et leur effet combiné sur la performance des entreprises.

\subsubsection{Quels sont les groupes technologiques de l'industrie 4.0?}

L'industrie 4.0 a été annoncée pour la première fois à la foire de Hanovre en 2011 pour marquer l'avènement de la 4ème révolution industrielle (Drath \& Horch, 2014). C'est un concept qui gagne de plus en plus de popularité dans les milieux industriels et académiques au point de devenir une mode. Comme conséquence, l'industrie 4.0 change de désignation et de définition d'un auteur à l'autre tout en restant centrée sur les technologies numériques. L'association allemande de télécommunication BITKOM révèle qu'il existe au moins 104 définitions différentes de l'industrie 4.0 (Bidet-Mayer, 2016). Au Québec, le Centre Facilitant la Recherche et l'Innovation dans les Organisations (CEFRIO) la définit comme étant un ensemble d'initiatives basées sur l'acquisition des données en temps réel qui permettent de prendre des décisions décentralisées en vue d'améliorer les processus, les produits et les services (CEFRIO, 2016). Comme on peut l'apercevoir à la figure 3.2, l'industrie 4.0 n'est que la continuité des grandes révolutions qui ont marqué la vie industrielle des trois derniers siècles. D'après le CEFRIO (2016), la première révolution industrielle correspond au passage de la production manuelle à la production mécanisée et ce vers la deuxième moitié du 18ème siècle. La seconde révolution industrielle à la fin du 19ème siècle se caractérise par l'électrification et la production en grande série. La troisième quant à elle, se caractérise par l'automatisation de la production grâce à l'électronique et les technologies de l'information dans les années 1960 - 1970. 


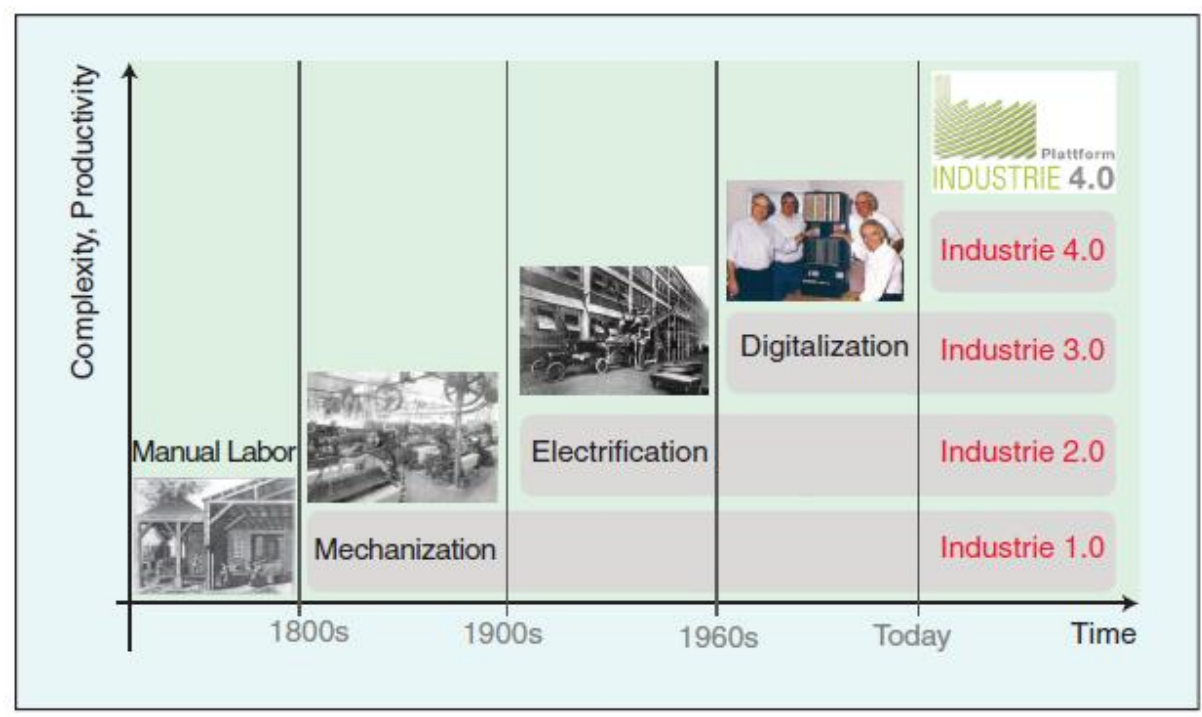

Figure 3.2. Évolution des systèmes de production depuis la première révolution industrielle. Adapté de (Drath \& Horch, 2014)

L'industrie 4.0 est un regroupement de plusieurs technologies numériques. Tout comme sa définition et sa désignation, les technologies rattachées à l'industrie 4.0 varient elles aussi d'un auteur à l'autre. Le tableau 3.4 qui suit est une synthèse de quelques publications entre 2015 et 2020 qui résume l'essentiel des différents groupes technologiques associés à l'industrie 4.0.

\begin{tabular}{|l|c|c|c|c|c|c|c|c|c|c|c|c|}
\hline & IoT & CPS & CC & BD & CS & MA & RA & SS & M2M & IA & IHV & FA \\
\hline (Dombrowski et al., 2017) & 1 & 1 & 0 & 1 & 0 & 1 & 0 & 0 & 1 & 0 & 0 & 0 \\
\hline (CEFRIO, 2016) & 1 & 1 & 1 & 1 & 1 & 1 & 1 & 1 & 1 & 1 & 0 & 0 \\
\hline (Gerbert et al., 2015) & 1 & 0 & 1 & 1 & 1 & 1 & 1 & 1 & 0 & 0 & 1 & 1 \\
\hline (Shahin et al., 2020) & 1 & 1 & 0 & 1 & 0 & 0 & 0 & 1 & 1 & 0 & 0 & 0 \\
\hline (Pagliosa et al., 2019) & 1 & 1 & 1 & 1 & 0 & 1 & 1 & 1 & 0 & 0 & 1 & 1 \\
\hline (Mayr et al., 2018) & 0 & 0 & 1 & 1 & 0 & 1 & 1 & 1 & 0 & 1 & 0 & 1 \\
\hline (Wagner et al., 2017) & 0 & 1 & 1 & 1 & 0 & 0 & 1 & 0 & 1 & 0 & 1 & 0 \\
\hline (Rossini, Costa, et al., 2019) & 1 & 0 & 1 & 1 & 0 & 1 & 1 & 0 & 0 & 1 & 0 & 1 \\
\hline (Kamble et al., 2020) & 1 & 0 & 1 & 1 & 0 & 1 & 1 & 0 & 0 & 0 & 0 & 1 \\
\hline
\end{tabular}

$1=$ Le groupe technologique fait partie de l'industrie 4.0 selon l'auteur

Tableau 3.4. Synthèse des groupes technologiques de l'industrie 4.0

L'application de la formule [1] au tableau 3.4 permet aussi d'obtenir la fréquence empirique de chaque groupe technologique de l'industrie 4.0 comme le montre le tableau 3.5 ci-dessous.

\begin{tabular}{|l|c|c|c|c|c|c|c|c|c|c|c|c|}
\hline & IoT & CPS & CC & BD & CS & MA & RA & SS & M2M & IA & IHV & FA \\
\hline Fréquence (\%) & 77.77 & 55.55 & 77.77 & 100 & 22.22 & 77.77 & 77.77 & 66.66 & 33.33 & 33.33 & 33.33 & 55.55 \\
\hline
\end{tabular}

Tableau 3.5. Fréquence empirique des groupes technologiques de l'industrie 4.0

À partir des tableaux 3.4 et 3.5, nous pouvons constater que l'internet des objets (IoT), les systèmes cyberphysiques (CPS), le cloud (CC), le big data (BD), les machines autonomes ou robots (MA), la réalité augmentée (RA), les systèmes de simulation (SS) et la fabrication additive (FA) 
sont les 8 principaux groupes technologiques de l'industrie 4.0. Ils sont les plus fréquemment identifiés par les auteurs dont les travaux vont de 2015 à 2020. Nous les retenons dans cet article en référence aux technologies de l'industrie 4.0. Néanmoins, il convient de mentionner que les autres groupes que sont l'intelligence artificielle (IA), l'intégration horizontale et verticale (IHV), la cyber sécurité (CS) et la communication machine to machine (M2M) sont indispensables pour certaines applications de l'industrie 4.0.

De tous ces groupes, une attention particulière est portée sur l'IoT que nous présentons un peu plus en détail à la section qui suit. C'est l'un des groupes technologiques les plus cités par les auteurs en référence à l'industrie 4.0. En plus, l'esquisse de solution que nous proposons dans cet article pour la promotion de la culture de la production épurée est essentiellement basée sur l'IoT.

\subsubsection{Description d'un système d'Internet des Objets}

L'IoT a débuté en 1999 dans le laboratoire AUTO-ID du MIT (Massachusetts Institute of Technology, 2020). La Radio Frequency Identification (RFID) aurait joué un rôle crucial pour l'invention de l'IoT (El Hakim, 2018). C'est grâce à elle que la transmission des informations d'identification des micropuces via un média sans fil est possible (Serpanos \& Wolf, 2018). Selon Benghozi (2009), l'IoT est un réseau des réseaux qui permet, via des systèmes d'identification électronique normalisés et unifiés, et des dispositifs mobiles sans fil, d'identifier directement et sans ambiguïté des entités numériques et des objets physiques et ainsi de pouvoir récupérer, stocker, transférer et traiter, sans discontinuité entre les mondes physiques et virtuels, les données s'y rattachant (Benghozi et al., 2009). Lorsque les objets sont connectés, ils forment un réseau dont les nœuds sont ces objets et ceux-ci peuvent en tout temps et en tout lieu échanger les données de nature variée. Il s'agit d'une forme d'ubiquité que confère cette technologie aux objets ce qui marque le principal avantage de l'IoT. Peu importe les lieux où se trouvent les objets et peu importe le moment, ceux-ci peuvent échanger les données grâce à l'IoT.

L'IoT est une infrastructure composée de systèmes qui interagissent ensemble afin d'atteindre les objectifs et les exigences spécifiques dans l'environnement où elle est déployée. Selon les applications, il peut y avoir plusieurs systèmes à tel point que l'infrastructure devient un système composé de plusieurs autres systèmes. C'est pourquoi le développement et la mise en œuvre d'une infrastructure IoT requièrent une architecture qui permet d'assurer un fonctionnement efficace en tenant compte des parties prenantes impliquées pour l'offre d'appareils, de systèmes de communication et réseaux, des fournisseurs de services et des développeurs d'affaires (Serpanos \& Wolf, 2018). Cette architecture dépend aussi des standards propres à chaque organisation. Dans la littérature, il existe des modèles architecturaux qui vont de 3 à 7 couches comme le montre la figure 3.3 .

En commun, tous les modèles architecturaux ont une couche de capteurs, une couche de réseaux et une couche d'application. La couche de capteurs désignée par "perception», «sensing», «physical» ou «environment » est celle qui est immédiatement en contact avec le monde physique. Elle permet de collecter les données dans l'environnement physique, d'identifier les objets de façon unique et d'établir la connectivité entre les appareils de nature différente (Gupta \& Quamara, 2018). Mais aussi, dans certains cas, elle peut servir pour véhiculer les commandes de l'infrastructure IoT aux actionneurs du monde physique. 


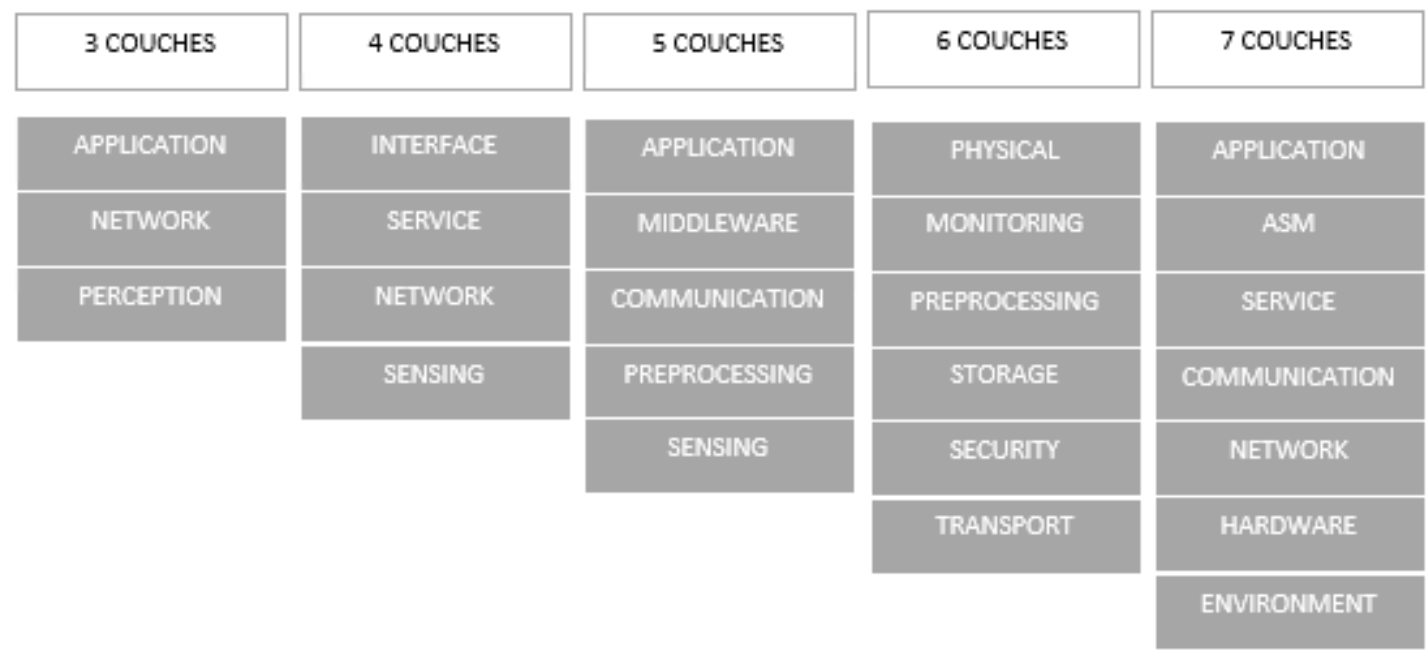

ASM = Application Support and Management

Figure 3.3. Modèles architecturaux des systèmes d'internet des objets (IOT). Tiré de (Sethi \& Sarangi, 2017), (Serpanos \& Wolf, 2018), (Gupta \& Quamara, 2018), et (Manoj Kumar \& Mallick, 2018)

La couche réseau ou «network» ou «transport» ou «communication » est aussi une couche que l'on retrouve dans tous ces modèles architecturaux. Elle a pour fonction d'assurer la transmission des informations entre les objets divers en utilisant spécifiquement le réseau internet (Sethi \& Sarangi, 2017). C'est probablement ce qui explique que ces systèmes soient labélisés comme étant les systèmes d'internet d'objets. On peut remarquer que le modèle à 7 couches a la particularité d'avoir à la fois une couche «network» et une couche «communication ». Dans ce modèle, la couche «communication » agit comme un pont qui établit le lien entre la couche des capteurs et la couche de service (Manoj Kumar \& Mallick, 2018) tandis que la couche «network» a la même fonction que celle des autres modèles architecturaux.

À l'exception du modèle à 6 couches, tous les modèles ont également en commun une couche « application » ou «interface » qui fournit à l'utilisateur final les informations utiles aux opérations et dans certains cas, lui permet d'interagir avec le système.

Les modèles à 4 et 7 couches se distinguent des autres par leurs architectures qui possèdent une couche de service. Essentiellement, cette couche sert de support pour les services spécifiques requis par les utilisateurs (Manoj Kumar \& Mallick, 2018), (Serpanos \& Wolf, 2018). Par exemple, l'exploitation d'un réseau électrique intelligent a des exigences de confidentialité spécifique et distincte de celle d'un système de gestion des péages (Serpanos \& Wolf, 2018). La couche de service dans l'une ou l'autre de ces applications offrira un service de confidentialité adapté. Les modèles à 5 et 6 couches possèdent quant à eux, une couche «preprocessing » qui ne se trouve pas dans les autres modèles. Au regard du volume et de la nature des données collectées par la couche des capteurs, il est nécessaire de conditionner celles-ci pour faciliter leur mise en réseau : c'est le rôle principal joué par la couche «preprocessing» (Sethi \& Sarangi, 2017).

La caractéristique propre au modèle à 5 couches est la couche «middleware ». Il s'agit en réalité d'une couche logicielle. Elle rend abstrait le monde physique qui est un monde réel et fournit un programme d'application de gestion des données à la couche finale, la couche d'application (Sethi \& Sarangi, 2017). Par exemple, dans le scénario de transport intelligent, la couche logicielle peut traiter les données collectées sur le trafic afin de prédire les conditions de circulation (X. Liu et al., 2019).

Le modèle à 6 couches possède une couche «monitoring », une couche «storage » et une couche «security» que l'on ne retrouve pas dans tous les autres modèles. Celles-ci contribuent au traitement très décentralisé des données, une particularité du modèle à 6 couches et jouent 
respectivement le rôle de gestion de la disponibilité des ressources, stockage des données sous différents formats et la confidentialité des données traitées (Manoj Kumar \& Mallick, 2018). Dans la littérature le modèle à 6 couches est aussi connu sous l'appellation «IoT fog-based architecture ». On peut remarquer que l'architecture modèle à 6 couches se limite à la couche «transport». Comme c'est un système très décentralisé, à chaque point de service une couche d'application peut être ajoutée après la couche «transport » pour offrir un service spécifique à l'utilisateur final.

Comparé aux autres modèles, celui à 7 couches possède les couches «hardware » et «ASM» qui sont propres à elle. La couche "hardware» permet d'intégrer tous les composants logiciels requis pour l'implémentation du système d'internet des objets (IoT) et la couche «ASM» permet d'avoir un contrôle de gestion complet du système y compris la sécurité et la protection des données (Manoj Kumar \& Mallick, 2018).

Nous pouvons constater que l'architecture de base d'un système IoT comprend nécessairement 3 couches à savoir une couche de capteurs, une couche de réseaux et une couche d'application. Cependant, il est assez complexe d'obtenir un modèle architectural standard des systèmes IoT car il faudrait considérer plus d'un milliard d'objets hétérogènes, les liaisons technologiques variées et plusieurs facteurs comme la sécurité, la confidentialité, la fiabilité, l'interopérabilité, les perspectives d'évolution du système, etc. (X. Liu et al., 2019). Toutefois, un écosystème IoT composé de 5 couches serait commun à plusieurs contextes d'application (El Hakim, 2018). Cette architecture est un bon compromis. En effet, lorsque les travaux de recherche se concentrent sur des aspects plus fins intégrant diverses technologies et une application à plusieurs paramètres, l'architecture à 3 couches n'est pas suffisante (Manoj Kumar \& Mallick, 2018). Celle à 7 couches est très développée pour des applications simples. Entre ces deux extrêmes, nous pensons qu'un système IoT classique devrait être composé de 5 couches, mais seule l'application finale devrait guider le choix du nombre de couches d'un système IoT.

\subsubsection{Complémentarité entre les technologies de l'industrie 4.0 et les pratiques de la production épurée}

Les technologies de l'industrie 4.0 ont toutes un dénominateur commun qui est la donnée numérique. En plus, elles se caractérisent par l'utilisation des moyens et des méthodes qui en fin de course permettent la collecte, l'analyse et la transmission de ces données entre les diverses parties impliquées dans la chaîne de création de valeur. Comme la donnée est présente à presque tous les maillons de cette chaîne, ces technologies trouvent ainsi une application presque illimitée. L'industrie 4.0 est présentée comme un facteur clé pour améliorer la productivité, promouvoir la croissance économique et garantir la durabilité des entreprises manufacturières (Rosin et al., 2020). Concrètement, l'un des avantages particuliers que l'industrie 4.0 apporte aux entreprises manufacturières est la capacité de s'adapter rapidement à des scénarios de demande volatile et des produits à court cycle de vie (Sanders et al., 2017). La demande des équipements de protection individuelle pour les hôpitaux pendant la crise du coronavirus en est un exemple de ce type de demande. Seuls les systèmes de production ayant une grande flexibilité et facilement reconfigurables ont pu répondre à cette demande. De façon plus générale, ces technologies permettent aux entreprises de réaliser les projets transformateurs sur le plan de la décentralisation, la virtualisation, l'interopérabilité, la modularité, l'orientation du service et l'ajustement de la capabilité en temps réel (M. Hermann et al., 2016).

Bien que ces technologies soient très pertinentes pour des transformations avantageuses des entreprises manufacturières, une étude renseigne que $50 \%$ des entreprises analysées affirment que la complexité de l'industrie 4.0 est un obstacle majeur à sa mise en œuvre (Dombrowski \& Richter, 2018). En plus, l'industrie 4.0 peut créer des situations qui rendent les entreprises trop dépendantes de la technologie au détriment de l'implication humaine ce qui peut à son tour réduire les habiletés humaines et la créativité nécessaires pour la résolution des problèmes et l'amélioration continue (Saito et al., 2012). Les interventions humaines dans une entreprise donnent une peu plus de "vie » 
aux activités de production et rendent la résolution des problèmes plus efficace. En effet, les machines et les systèmes ne peuvent s'autoaméliorer; une main-d'œuvre engagée et capable de créativité lors de la résolution des problèmes est essentielle pour une amélioration continue durable (Maginnis et al., 2020). Il serait donc illusoire de penser que les technologies de l'industrie 4.0 prises isolément peuvent résoudre tous les problèmes auxquels font face les entreprises manufacturières. Les solutions numériques ne facilitent que la collecte, le traitement et la transmission des données. D'après Rüttimann (2016), les initiatives portées par l'industrie 4.0 sont potentiellement vouées à l'échec tant qu'elles ne prennent pas en considération les principales lois de la production épurée (Rüttimann \& Stöckli, 2016). Si les pratiques de la production épurée sont médiocres dans une entreprise, l'introduction de ces technologies n'aura qu'un effet amplificateur de la médiocrité existante. Ainsi, l'expression "garbage in garbage out » s'applique aussi aux technologies de l'industrie 4.0 et marque la principale limite de ce nouveau concept en production industrielle.

Les pratiques de la production épurée quant à elles ont déjà prouvé leur contribution à la performance des entreprises dans divers secteurs d'activités. Notamment, il a été montré par plusieurs chercheurs que ces pratiques contribuent à la performance opérationnelle, financière et environnementale des entreprises (Taj \& Morosan, 2011), (Dora et al., 2013), (Panwar et al., 2018), (Yang et al., 2011), (Nawanir et al., 2013), (Hofer et al., 2012), (Fullerton et al., 2014), (GarzaReyes et al., 2018), (Kashav et al., 2019). Cependant, il semble que ces pratiques à elles seules ne soient plus capables de faire face à la dynamique actuelle du marché (Kolberg et al., 2017). Tout comme les technologies de l'industrie 4.0, les pratiques de la production épurée ont donc aussi des limites et probablement, n'arriveront pas à répondre aux demandes du futur.

De plus en plus, l'idée selon laquelle l'industrie 4.0 et la production épurée peuvent coexister et se compenser mutuellement pour l'atteinte de leur objectif commun qui ultimement est l'optimisation et l'autonomisation des opérations manufacturières est mise en avant. À cet effet, Rosin (2020) a synthétisé des travaux qui montrent que le JIT l'une des 5 méthodes de la production épurée, peut être supportée par l'IoT, le BD, le CC, la SS, les MA. L'une des principales caractéristiques du JIT est la production à flux tirés. Le kanban, une des techniques qui se rattache au JIT, permet de gérer les approvisionnements des postes de travail et de contrôler la cadence des opérations contribuant ainsi à la production à flux tirés. Un des exemples concrets du lien de complémentarité entre les pratiques de production épurée et les technologies de l'industrie 4.0 est la numérisation du kanban qui devient le eKanban comme l'indiquent les travaux synthétisés par Rosin (2020).

Le HRM est aussi un exemple des méthodes de la production épurée qui peut s'opérationnaliser en complément avec les technologies de l'industrie 4.0. L'introduction de ces technologies impulse nécessairement une nouvelle dynamique entre les employés et les systèmes de production. D'après Davies (2017), les opérationnels de première ligne dans un environnement de production 4.0 ne sont plus des agents passifs qui accomplissent leurs tâches sans références ; au contraire, ils sont élevés au statut valorisant de "knowledge worker » (Davies et al., 2017). Grâce à la technologie, les employés se trouvent ainsi mis en valeur ce qui est un facteur de motivation. En plus, comme les technologies de l'industrie 4.0 facilitent la décentralisation des prises de décision, elles contribuent ainsi à la responsabilisation des employés. La valorisation et la responsabilisation des employés qui sont recherchés par le HRM, sont portées par les technologies de l'industrie 4.0. Ceci illustre la complémentarité entre la production épurée et l'industrie 4.0.

Plus généralement, l'industrie 4.0 offre un ensemble de solutions technologiques qui numérisent et soutiennent les pratiques classiques de la production épurée comme c'est le cas avec le JIT et le HRM dans les exemples ci-dessus mentionnés. Historiquement l'industrie 4.0 a été précédée par la production épurée. Ce nouveau concept peut être considéré comme une évolution logique des principes de la production épurée et l'aide à réaliser son plein potentiel (Sanders et al., 2017). La 
mise en oeuvre de la production épurée n'exclut pas la mise en œuvre de l'industrie 4.0. Les deux ont une relation de complémentarité comme l'affirment au moins 2 travaux récents (Buer et al., 2021), (Ciano et al., 2021). Dans la pratique, il est judicieux de prioriser l'implémentation de la production épurée avant un passage à la numérisation des méthodes et outils de travail dans une entreprise (Staufen AG, 2015).

\subsubsection{Effets combinés de l'industrie 4.0 et de la production épurée sur la performance opérationnelle des entreprises}

La performance opérationnelle d'une entreprise manufacturière traduit son habileté à rapidement réussir les mises en production de qualité qui correspondent aux besoins changeants des clients et ceci avec des moyens limités. Cette performance opérationnelle se mesure par quelques indicateurs tels que le coût, la vitesse, la qualité et la flexibilité (Slack et al., 2013). Les technologies de l'industrie 4.0 opèrent sur le principe de l'intégration horizontale et verticale des systèmes manufacturiers et sont basées sur l'échange des données en temps réel entre les partenaires variés de la chaîne de valeur (Kamble et al., 2020). Cette possibilité d'échange et de traitement des données en temps réel et l'interconnectivité de tous les acteurs de la chaîne de valeur qu'offrent les technologies de l'industrie 4.0 ont un effet de levier sur les pratiques existantes de la production épurée. Elles propulsent la performance opérationnelle que la production épurée apporte aux entreprises. À cet effet, une enquête effectuée par Rossini (2019) auprès de 108 entreprises manufacturières européennes renseigne que quel que soit le contexte, la production épurée combinée à l'industrie 4.0 améliore grandement la performance opérationnelle des entreprises européennes (Rossini, Costa, et al., 2019). À cette enquête, s'ajoute celle effectuée au Brésil par Tortorella (2018) auprès de 110 entreprises manufacturières (Tortorella \& Fettermann, 2018). Il a montré après une analyse des données par les statistiques multivariées que même si les conditions socioéconomiques sont défavorables dans les pays en voie de développement, les pratiques de la production épurée sont positivement liées aux technologies de l'industrie 4.0 et cette liaison aboutie au sens large, à une plus grande amélioration des performances des entreprises.

En plus de la propulsion de la performance opérationnelle, l'industrie 4.0 a un effet sur la pérennisation de cette performance. Celle-ci correspond au maintien de la performance sur le long terme c'est-à-dire la performance durable des entreprises. Cela a été montré par le travail effectué par Kamble (2020) qui indique que les technologies de l'industrie 4.0 superposées aux pratiques de la production épurée ont des effets directs et indirects sur la performance durable des entreprises malgré le fait que celles-ci évoluent dans un environnement défavorable (Kamble et al., 2020). Ce résultat a été obtenu par les données collectées auprès de 115 entreprises manufacturières du secteur automobile, chimique et pharmaceutique de l'Inde et analysées avec par le test de KolmogorovSmirnov et de Harman. En plus du travail de Kamble, Muhammad (2021) a montré à l'aide d'un cas d'étude qu'il est possible de combiner la production épurée, l'industrie 4.0 et la production verte pour rapidement améliorer la performance environnementale des entreprises qui en soit est une performance durable (Amjad et al., 2021). Les travaux effectués par Tortorella (2018), Rossini (2019), Kamble (2020) et Muhammad (2021) peuvent tout au moins nous laisser penser que l'introduction des technologies de l'industrie 4.0 au sein des entreprises manufacturières n'est pas nuisible aux pratiques de la production épurée. Que l'on soit dans un environnement socioéconomique favorable ou non et peu importe le secteur d'activité, l'effet conjugué de ces deux concepts est l'amélioration rapide de la performance opérationnelle des entreprises sur le long terme.

\subsubsection{Quel groupe technologique de l'industrie 4.0 pour quel groupe méthodologique de la production épurée?}

Des propositions et des cas de projets réalisés dans la littérature permettent d'établir les associations possibles entre les technologies de l'industrie 4.0 et les méthodes de la production 
épurée. Pagliosa (2019) a présenté 52 exemples d'application de 9 technologies de l'industrie 4.0 à diverses pratiques de la production épurée (Pagliosa et al., 2019). Dans ces exemples, l'internet des objets (IoT), les systèmes cyberphysiques (CPS), le big data (BD), les systèmes d'intégration (IHV) et les systèmes de simulation (SS) sont applicables à tous les niveaux de la chaîne de valeur. Rosin (2020) a compilé des publications entre 2009 et 2019 qui montrent que 7 technologies de l'industrie 4.0 dont, l'internet des objets (IoT), les systèmes de simulation (SS), le big data (BD), le cloud (CC), les robots (MA), les systèmes d'intégration (IHV) et la réalité augmentée (RA) peuvent s'appliquer aux pratiques fondamentales de la production épurée que sont la gestion des ressources humaines, la chasse aux gaspillages, le Juste-à-Temps et le Jidoka (Rosin et al., 2020). En plus de présenter un cas concret d'application des technologies de l'industrie 4.0 à la gestion des en cours de production, Shahin (2020) quant à lui a identifié des cas d'études concrètement réalisées en contexte de production épurée pour lesquels, le big data (BD), le cloud (CC) l'internet des objets (IoT) sont mis à contribution (Shahin et al., 2020).

Ces travaux confirment d'abord que les technologies de l'industrie 4.0 sont combinables aux pratiques de la production épurée. Ensuite ils permettent comme le présente le tableau 3.4, de faire un bilan des liaisons possibles entre les groupes technologiques de l'industrie 4.0 et les groupes méthodologiques de la production épurée. Nous pouvons remarquer que l'internet des objets (IoT), les systèmes cyberphysiques (CPS), le cloud (CC), le big data (BD), la réalité augmentée (RA) et les systèmes d'intégration (IHV) sont les technologies de l'industrie 4.0 les plus utilisées pour la numérisation des groupes méthodologiques de la production épurée.

\begin{tabular}{|l|c|c|c|c|c|}
\hline & JIT & TPM & TQM & CI & HRM \\
\hline IoT & $\bullet$ & $\bullet$ & $\bullet$ & & \\
\hline CPS & $\bullet$ & $\bullet$ & $\bullet$ & & \\
\hline CC & $\bullet$ & $\bullet$ & $\bullet$ & $\bullet$ & \\
\hline BD & $\bullet$ & $\bullet$ & & $\bullet$ & \\
\hline MA & $\bullet$ & & & & \\
\hline RA & $\bullet$ & $\bullet$ & $\bullet$ & $\bullet$ & \\
\hline SS & $\bullet$ & & & & \\
\hline M2M & $\bullet$ & $\bullet$ & & & \\
\hline IHV & $\bullet$ & & $\bullet$ & $\bullet$ & \\
\hline FA & $\bullet$ & $\bullet$ & & & \\
\hline
\end{tabular}

Tableau 3.4. Quelles technologies 4.0 pour quelles méthodes de la production épurée?

\subsection{Production épurée et comportement des équipes de travail}

\subsubsection{Comportement des équipes de travail}

Dans la structure de nombreuses organisations, la cellule de base est l'équipe de travail et non l'individu (Rousseau et al., 2006b). Une équipe de travail est un ensemble formel et permanent d'au moins deux individus interdépendants et collectivement responsables de l'accomplissement d'une ou de plusieurs tâches définies par l'organisation (Rousseau et al., 2006a). La qualité de l'interaction et l'ensemble des valeurs communes au sein des équipes de travail d'une entreprise manufacturière contribuent à l'atteinte des objectifs de production.

Ainsi, il serait difficile d'envisager 
l'opérationnalisation de la production épurée dans les entreprises en faisant fi du comportement ou de la culture qui règne dans les équipes de travail. Ce comportement évolue en fonction des phases du cycle de vie de l'équipe.

D'après le modèle de Tuckman (1965), le cycle de vie d'une équipe comprend quatre phases à savoir la phase de formation, de turbulence, de normalisation et de performance (Tuckman, 1965). Ce modèle est semblable à celui de Schermerhorn (2014) qui comprend aussi quatre phases soit la phase de constitution, de tumulte, de cohésion et de rendement. À la phase de formation ou de constitution, chaque membre commence à s'identifier à d'autre membre et à l'équipe elle-même (Schermerhorn et al., 2014). La phase de turbulence ou de tumulte est caractérisée par une dimension transitoire au cours de laquelle les membres de l'équipe s'entrechoquent en vue de former un équilibre dans les rôles et les règles de fonctionnement. Cette phase est riche en tensions et en émotions pour ses membres (Schermerhorn et al., 2014) par conséquent, il est plus difficile pour eux de coordonner leurs actions et adapter leurs comportements aux exigences de la tâche et aux autres membres de l'équipe (Fiore et al., 2001). À la phase de normalisation ou de cohésion, la résistance entre les membres est vaincue le sentiment d'intégration et la cohésion se développent; de nouvelles normes comportementales évoluent et de nouveaux rôles sont adoptés (Tuckman, 1965). Après la normalisation, l'équipe atteint la maturité, devient bien organisée et opérationnelle (Schermerhorn et al., 2014). C'est la phase de rendement ou de performance.

Les milieux de travail sont souvent hétérogènes. Dans diverses entreprises, ces phases ne peuvent pas nécessairement toutes se manifester dans le cycle de vie de toutes les équipes de travail. En plus, d'une équipe à l'autre, elles ne s'étalent pas nécessairement sur la même durée. Cependant, le comportement des membres d'une équipe de travail est reconnaissable à partir de son niveau de maturité qui correspond à la phase où elle se situe dans son cycle de vie. Peu importe le niveau de maturité d'une équipe, les comportements que les membres manifestent entre eux facilitent la réalisation des tâches communes (Rousseau et al., 2006a). Ceux-ci seront plus ouverts aux pratiques de création de valeur dans une entreprise et enclin à proposer les idées d'amélioration lorsque les comportements des membres à leur égard sont favorables ou lorsque la culture organisationnelle prônée par le leadership démontre une forme de respect et de confiance à leur égard. Le comportement des équipes de travail peut donc faire ou défaire l'opérationnalisation de la production épurée. De toute évidence, il est plus facile pour une équipe qui est dans sa phase de normalisation ou de performance d'adopter les pratiques de la production épurée que pour une équipe qui est dans sa phase de turbulence ou de tumulte.

Rousseau (2006) a déterminé 7 dimensions du fonctionnement interne des équipes de travail que sont la coopération, la communication, le soutien psychologique, la gestion de conflits, la planification/organisation du travail, la gestion des ressources et le soutien à l'innovation indispensables à des degrés différents au rendement des équipes de travail (Rousseau et al., 2006a). Emiliani (1998) quant à lui a introduit le concept de «lean behavior » pour désigner un ensemble de comportements qui facilite l'opérationnalisation de production épurée. Parmi ceux-ci, il y a par exemple la bienveillance, l'humilité, la compassion, la déférence, le calme, la quiétude, l'honnêteté, la cohérence, la générosité, la patience, l'humour, le respect, l'écoute, la confiance, la sincérité, l'objectivité et la discipline (Emiliani, 1998). Les méthodes et les outils techniques tout seuls sont insuffisants pour réussir à opérationnaliser la production épurée au sein d'une entreprise même si ceux-ci sont accompagnés par les technologies de l'industrie 4.0. La personne humaine demeure au centre de toutes les actions. En conséquence, aux outils techniques il est indispensable d'associer dans le cycle de vie des équipes de travail, des comportements qui sont portés par la culture d'entreprise et la culture locale des équipes de travail. Ces comportements peuvent être développés en agissant sur des facteurs que nous présentons à la section qui suit. 
Un comportement humain dans un milieu de travail est la résultante de plusieurs facteurs qui sont à la fois sous le contrôle et hors du contrôle de la personne qui manifeste ce comportement. Le behaviorisme, la psychologie cognitive, la psychothérapie systémique et l'économie comportementale sont les principales approches de la psychologie qui permettent de comprendre les mécanismes ou les facteurs qui déterminent la formation d'un comportement (Kinley \& Ben-Hur, 2015).

Le behaviorisme est une approche en psychologie qui a été vulgarisée par Watson en 1913 (Wertheimer \& Puente, 2020), mais qui a commencé avec les travaux de Pavlov publiés en 1897 sur l'étude du comportement des chiens. D'après cette approche, la réponse aux stimuli qui environnent une personne façonne ses actions et donc son comportement (Georgia State University, 2017). Ainsi, pour comprendre la formation d'un comportement, il suffit d'identifier comme l'indique Kinley (2015), les stimuli, les conditions nécessaires et les incitatifs qui sont à l'origine du comportement. Par opposition aux évènements internes tels que les sentiments, le behaviorisme est basé sur les faits externes qui sont observables et mesurables. Tout comportement, aussi complexe soit-il peut être réduit à une simple relation stimulus-réponse (McLeod, 2017). Un peu comme dans une relation cause-effet, le stimulus est la cause et le comportement est l'effet. Cette approche promeut une démarche scientifique à la compréhension du comportement humain (Graham, 2019). De ce fait, il est possible de prédire le comportement d'une personne ou déterminer les stimuli qui engendreront un certain comportement de celle-ci.

Les «soft practices » de la production épurée directement rattachés aux «lean behavior», ont la particularité de ne pas être observables et mesurables de manière objective. Par exemple le degré de respect, de confiance ou de bienveillance d'une personne varie d'un observateur à l'autre. Leur nature est qualitative. Or la démarche du behaviorisme est scientifique et objective. De ce fait, il y a une inadéquation entre l'approche du behaviorisme et les «lean behavior». En plus, il est difficile de prédire un «lean behavior» en établissant une relation cause-effet comme le promeut le behaviorisme car ces comportements sont pour la majorité des cas des valeurs humaines et par nature les humains sont des êtres instables. Le behaviorisme nous semble donc moins indiqué pour promouvoir et gérer efficacement les «soft practices » de la production épurée.

La psychologie cognitive renvoie à l'étude des processus mentaux internes; toutes choses qui se passent à l'intérieur du cerveau humain y compris la perception, la pensée, la mémoire, l'attention, le langage, la résolution de problèmes et l'apprentissage (Cherry, 2019). À la différence du behaviorisme, la psychologie cognitive perçoit un comportement comme étant le résultat d'un processus de réflexion délibéré et conscient (Kinley \& Ben-Hur, 2015). C'est ce qui se passe à l'intérieur de la personne et non dans l'environnement externe de celle-ci qui est à l'origine de son comportement. Avec cette approche, il est plus beaucoup question d'identifier comme le mentionne Valentin (2012), les processus mentaux même inconscients qui s'intercalent entre les stimuli et la réponse (Valentin et al., 2012) pour comprendre la formation d'un comportement. Entre les stimuli et la réponse, on trouve essentiellement les intentions de la personne qui sont la combinaison des normes sociales, ce que la personne pense que les autres font et considéré comme normal, les attitudes et la perception de la personne (Kinley \& Ben-Hur, 2015).

Emiliani (1998) présente les «lean behavior» comme le fait de minimiser les gaspillages dont les origines sont les pensées arbitraires ou contradictoires qui aboutissent à des comportements défensifs, des relations inefficaces, une pauvre coopération et des attitudes négatives (Emiliani, 1998). Concrètement, les «lean behavior » renvoient aux savoir-être (comportements et attitudes) qui facilitent la production épurée. D'après le modèle tripartite de Rosenberg (1960), les attitudes ont une composante cognitive constituée de l'ensemble des croyances, des opinions, des connaissances et de l'information que possède une personne; une composante affective constituée des sentiments particuliers qu'éprouve une personne à l'égard des autres et une composante conative 
soit l'intention ou la prédisposition à agir d'une façon donnée (M. J. Rosenberg, 1960), (Schermerhorn et al., 2014). La gestion des attitudes ou des savoir-être et donc des "lean behavior » nous semble être plus indiquée avec l'approche de la psychologie cognitive. Le centre d'intérêt de cette approche est ce qui se passe à l'intérieur de la personne. Cela correspond aux composantes cognitive et affective des attitudes. En plus, les méthodes d'analyse de cette approche sont appropriées pour des phénomènes tels que les processus mentaux internes qui tout comme les «lean behavior », sont difficilement observables et mesurables avec objectivité. Les critiques formulées dans la littérature sur les méthodes non rigoureuses de la psychologie cognitive deviennent plutôt des avantages pour son application à la gestion des «lean behavior».

La psychothérapie systémique se base sur la nature des relations et des interactions entre la personne et son environnement pour expliquer son comportement (Kinley \& Ben-Hur, 2015). La personne humaine et son environnement forment un système. Dans une entreprise, le système dans lequel évolue une personne est principalement composé des collègues, des moyens et des méthodes de travail, mais aussi de son espace de travail et de la matière qu'il transforme. Le comportement adopté par cette personne est alors déterminé à chaque instant par la nature de la relation entre elle et les éléments de ce système. La psychothérapie systémique a émergé avec les travaux de recherche de Bateson vers les années 1950 (Borcsa \& Stratton, 2016) et trouve des applications aussi bien en thérapie individuelle qu'en thérapie de groupe.

Dans une entreprise, les équipes de travail peuvent être considérées comme des sous-systèmes sous l'influence de la culture d'entreprise et la culture propre à chaque équipe. Puisque l'approche de la psychothérapie systémique est basée sur la nature des relations et interactions entre les composants d'un système, l'un des mécanismes ou facteurs pour gérer les comportements consiste à comprendre le sens tel qu'il est créé dans le langage ce qui permet d'identifier les pensées et les croyances de chaque personne et les relier à leurs sentiments, afin de créer un nouveau sens et la possibilité de relations alternatives (Gergen, 1982), (Lock \& Strong, 2010), (McNamee \& Gergen, 1992). La psychothérapie systémique pourrait être appliquée en gestion des «lean behavior» par le développement d'un environnement de travail qui facilite ce type de comportement.

L'approche de l'économie comportementale quant à elle, est relativement récente et tire avantage des sciences économiques et de la psychologie pour explorer la manière que les personnes se comportent. Cette approche repose sur la théorie des perspectives selon laquelle les choix des personnes s'opèrent suivant un système dual de pensée (Kahneman, 2011). D'un côté (système 1), les choix se font rapidement, automatiquement, avec peu ou pas d'effort et aucune volonté de contrôle (Hayes, 2020). De l'autre côté (système 2), les choix se font de manière délibérée, lente et plus logique (Kahneman, 2011). Au sein d'une même personne, ces deux systèmes de décision coexistent. Comme les choix opérés par le système 1 se font avec peu ou pas d'effort et aucune volonté de contrôle, ils sont très souvent différents de ceux opérés par le système 2 . Face à une même situation, un petit détail apparemment insignifiant dans l'environnement des personnes peut avoir un impact majeur sur sa décision et donc son comportement (Thaler \& Sunstein, 2009). L'idée de cette approche est de développer des «nudge » adaptés à l'environnement dans lequel le système 1 est prédominant qui aident à surmonter les biais connus de sorte que les décisions automatiques et rapides soient alignées avec les décisions lentes et délibérées (Brown et al., 2020). Les "nudge » sont ces détails qui permettent différentes influences (psychologiques, culturelles et sociales) sur la prise de décision et le comportement des états, des collectivités, des entreprises et des individus (Singler, 2015).

D'après Singler (2015), il existe trois catégories de facteurs qui nous influencent dans la prise de décision. Il s'agit des facteurs sociaux qui font référence à l'influence qu'ont les autres sur nous et ce de manière consciente ou non; des facteurs situationnels en référence à la manière dont l'information et les options nous sont présentées dans l'environnement décisionnel et des facteurs personnels qui eux sont liés à notre propre histoire, à nos émotions et à nos biais (Côté, 2018) . Les 
«nudge » peuvent être taillés sur mesure pour agir sur une combinaison de ces facteurs et ainsi influencer les prises de décisions. L'influence sociale des autres personnes peut agir les attitudes et modifier les savoir-être d'une personne afin de se conformer à l'équipe. Pour cela, il suffit par exemple qu'un des membres d'une équipe de travail agisse comme " nudge » et l'approche de l'économie comportementale trouve une application dans la gestion des «lean behavior». En plus des facteurs sociaux, les facteurs situationnels peuvent être exploités pour changer au moins un des "lean behavior» qu'est l'honnêteté. Pour cela, Gino (2017) a effectué des expériences de terrain qui montrent qu'un changement sur l'emplacement de la signature d'un document prépare les personnes à faire des déclarations honnêtes (Gino, 2017). Cependant, l'approche de l'économie comportementale est basée sur le système de prise de décision. Cela signifie que si la composition d'une équipe de travail, son organisation et sa charge de travail sont telles que les membres de cette équipe décident dans la majorité des cas avec leurs systèmes 2, alors 1'introduction des "nudge » sera sans effet sur leur comportement. Ces éléments nous amènent à dire que l'exploitation de l'économie comportementale pour la gestion des «lean behavior» dépend du contexte d'application. Dans certains cas il est possible de mettre à contribution l'économie comportementale à cette fin et dans d'autres cas cela est simplement impossible.

En conclusion, à la base d'un comportement humain se trouvent un ou plusieurs facteurs qui peuvent être les stimuli, les incitatifs, les conditions ou contexte environnants, les attitudes, les normes sociales, les perceptions, les interactions entre la personne et le système sociotechnique et les modes de prise de décision de la personne. Ces facteurs peuvent aussi être interprétés comme des événements qui impactent le comportement d'une équipe de travail car une équipe est composée de personnes humaines. Mais aussi, le niveau de maturité d'une équipe qui dépend de la durée de vie de celle-ci, impacte aussi le comportement d'une équipe de travail comme l'on indiqué les modèles de Tuckman (1965) et de Schermerhorn (2014). Donc les évènements introduits de l'extérieur ou de l'intérieur à des moments de la vie d'une équipe déterminent la manifestation de son comportement. En agissant sur les facteurs ci-dessus identifiés, il serait possible de mieux gérer les «lean behavior» ou comportements favorables à la production épurée dans une équipe de travail. Les approches qui semblent plus indiquées à cette fin sont la psychologie cognitive et la psychothérapie systémique. Le behaviorisme et l'économie comportementale seraient plus appropriés pour la gestion du passage aux actions favorables à la production épurée.

\subsection{Potentiels des technologies pour le changement comportemental}

De l'invention de l'automobile à la vulgarisation ou des téléphones portables, l'accès aux nouvelles technologies a toujours eu un impact sur les comportements des personnes ou même de la société dans son ensemble. L'arrivée des technologies de l'industrie 4.0 dans le secteur manufacturier n'aura pas seulement un effet sur la modernisation des outils de production, mais très probablement sur les comportements des personnes impliquées dans les opérations quotidiennes. Dans le domaine des sciences sociales, l'émergence de ces technologies a impulsé le concept de «Behavior Change Technology» dont le but est de développer des systèmes spécifiques pour l'adoption des comportements ciblés (Productive Edge Team, 2015) et des travaux de recherchent renseignent que ces technologies peuvent jouer un rôle d'amplificateur des comportements déjà existants, de promotion des nouveaux comportements ou de maintien de la dynamique de changement comportemental (Joinson \& Piwek, 2013), (Lilly \& Durr, 2012), (Locke, 2009), (Cascio \& Montealegre, 2016).

\subsubsection{Potentiel amplificateur des comportements déjà existants}

Tout d'abord, ces technologies sont désignées par Fogg (2003) par l'expression «Technologies Persuasives » et il les définit comme tout système informatique interactif conçu pour changer l'attitude et le comportement des personnes (Fogg, 2003). D'après lui ces technologies sont à la fois un outil de travail, un média, mais aussi un acteur social (Michaud, 2014). En tant qu'acteur social, les technologies numériques peuvent être considérées comme des membres à part entière des 
équipes de travail. En effet, Reeves (1996) a montré que les humains réagissent inconsciemment aux médias de la même manière qu'ils répondent aux autres humains (Soash, 1999).

$\mathrm{Au}$ sein des équipes de travail, ces nouveaux acteurs peuvent être dimensionnés pour accompagner une démarche de changement comportemental. Elles peuvent supporter l'application des approches de la psychologie cognitive, du behaviorisme, de l'économie comportementale ou de la psychothérapie systémique. Le potentiel d'amplification des comportements qu'ont les technologies provient de leur capacité à transcender les limites cognitives, comme la mémoire dans les activités de réflexion d'apprentissage et de résolution de problèmes (Pea, 1985). En général, les objets ou les environnements comme l'affirme le psychologue cognitiviste Gibson (1979), ont certaines propriétés qui conduisent à différents types de comportements (Gibson, 1979). Essentiellement, les objets technologiques servent à amplifier les intentions et les actions des personnes (Toyama, 2015). Si les choses que les gens essaient de faire sont utiles et bien adaptées au public servi, la technologie pourra amplifier leurs efforts positifs (Toyama, 2015). Mais si ce que les gens veulent faire n'est pas positif, la technologie pourra aussi bien amplifier leurs mauvaises intentions (Toyama, 2015). Par exemple, certaines études qui ont examiné les effets de la télévision câblée et des médias sociaux sur les attitudes politiques et sociales ont permis de conclure que le design des technologies et les choix que les personnes font peuvent amplifier leurs valeurs déjà existantes (Schoemaker, 2014).

\subsubsection{Potentiel de promotion des nouveaux comportements}

La capacité que possèdent les objets technologiques d'attirer ou de séduire leur confère un potentiel de promotion des nouveaux comportements. De ce fait, un objet visuellement attractif aux yeux de l'utilisateur a plus de chance d'influencer les comportements de ce dernier (Michaud, 2014). Fogg (2003) a énoncé 13 principes qui expliquent comment les objets technologiques mobiles ont une opportunité unique de persuasion. Ces principes montrent que ces objets peuvent à la fois promouvoir et maintenir une dynamique de changement comportemental. Parmi ces principes, il y a par exemple le principe de la facilitation sociale selon lequel les gens sont plus susceptibles d'adopter un comportement ciblé s'ils savent qu'ils sont observés via la technologie, ou s'ils peuvent discerner via la technologie que d'autres exécutent ce comportement avec eux (Fogg, 2003), peut contribuer à promouvoir de nouveaux comportements au sein d'un groupe. Lorsqu'un système est semblable aux utilisateurs, il a plus de chance d'influencer ceux-ci d'une quelconque façon puisqu'ils pourront s'identifier à ce système (Michaud, 2014). Essentiellement, le potentiel de promotion de nouveaux comportements des technologies persuasives repose sur les 13 principes de Fogg (2003). Un exemple d'application d'une technologie de cette nature est l'utilisation des médias sociaux comme moyen de promotion des comportements alimentaires sains en vue d'une perte de poids (Jane et al., 2015).

\subsubsection{Potentiel de maintien de la dynamique de changement comportemental}

En changement comportemental, il est parfois un plus facile d'amorcer le changement, mais maintenir et consolider ce changement représentent souvent un véritable défi (Johnson, 2013). L'un des objectifs des technologies numériques est de créer un espace favorable qui relie les personnes, les ordinateurs, les réseaux et les objets, surmontant ainsi les limites du monde physique et de l'espace numérique (Cascio \& Montealegre, 2016). Lorsque cet objectif est atteint, il permet de vivre de nouvelles expériences dans un groupe de travail. Les idées qui en résultent de ces expériences permettront aux organisations de créer un changement de comportement plus convaincant et donc durable (Lake, 2018). La connectivité qu'offrent les technologies numériques donne aussi la possibilité de partager plus facilement les expériences diverses et sert de support pour véhiculer en permanence la culture de l'entreprise et la culture locale des équipes de travail. Ce partage d'expériences en continu créer en retour une ambiance de soutien mutuel entre les membres d'une équipe et confère à la technologie un potentiel exploitable pour le maintien de la dynamique de changement comportemental. De ce fait, la technologie assure la maintenance des comportements. 
Un exemple de cette application est celui de Capital One qui ayant voulu accroître la collaboration entre les membres de ses équipes, a utilisé une plateforme logicielle de communication et de collaboration dans chacun de ses départements pour ainsi permettre aux employés d'avoir des interactions en temps réel, le partage d'idées et des présentations (Pamela, 2018).

Nous pouvons constater qu'en plus de la dimension technique c'est-à-dire la numérisation des outils techniques et des méthodes de la production épurée, les technologies numériques peuvent aussi être exploitées pour la dimension non technique c'est-à-dire la gestion des "lean behavior ». En tant que véritable acteur social, nouveau membre des équipes de travail, ces technologies peuvent servir de véhicule de la culture d'entreprise et de point d'appui pour promouvoir de nouveaux comportements, amplifier les comportements ciblés ou maintenir une dynamique de changement comportemental.

Cependant, les différentes perceptions qu'on les personnes sur l'introduction de ces technologies dans leurs milieux de travail, peut miner l'acceptation sociale de celles-ci. En effet, l'introduction des nouveaux systèmes technologiques dans les milieux de travail peut accroître le sentiment d'être surveillé et le sentiment d'oppression ce qui engendrerait l'insatisfaction, la démotivation et à long terme le mal-être des personnes (Van Acker et al., 2019). À cause de ces sentiments négatifs, les personnes auront une tendance répulsive à l'égard de ces technologies. Il a été aussi suggéré que l'introduction de ces systèmes pourrait entraîner un manque d'autonomie et un déficit de compétences qui conduiront par la suite aux comportements contre-productifs (Cascio \& Montealegre, 2016). Cependant, le travail de Van Acker (2019) montre que lorsque le cadrage de ces technologies vise les objectifs intrinsèques comme améliorer la santé et favoriser l'autonomie, cela aboutit à plus d'acceptabilité que lorsque le cadrage de celles-ci vise les objectifs extrinsèques comme l'accroissement de la productivité et la surveillance (Van Acker et al., 2019). Il est donc judicieux d'anticiper sur l'acceptabilité sociale des nouvelles technologies lorsqu'on souhaite les mettre au service du changement comportemental au sein des entreprises.

Au-delà du défi de l'acceptabilité sociale, il se pose aussi l'enjeu de la santé psychologique des employés qui peut être remise en cause avec l'introduction de ces technologies dans leurs milieux de travail. L'Organisation mondiale de la santé (OMS) définit la santé psychologique comme un état de bien-être dans lequel une personne peut se réaliser, surmonter les tensions normales de la vie, accomplir un travail productif et contribuer à la vie de sa communauté (ASPAM, 2021). Or certains travaux ont montré que le stress et l'anxiété reliés à la technologie peuvent avoir des effets négatifs sur les personnes et les résultats des organisations (Meuter et al., 2005), (Kummer et al., 2017). L'anxiété numérique se manifeste par des sensations de tension et d'inconfort par rapport à l'émergence et l'intégration de nouvelles technologies dans le milieu de travail (Pfaffinger et al., 2020). Très clairement cette anxiété est un problème de santé psychologique. Elle peut être déclenchée par des facteurs sociétaux, organisationnels et individuels comme par exemple le défi d'adaptation à la technologie, les attentes trop élevées des organisations ou les changements introduits dans les méthodes de travail qui provoque la résistance au changement (Pfaffinger et al., 2020). Comme conséquences, la dépression des employés et l'accroissement des sentiments négatifs reliés au syndrome du survivant mentionné par Ouimet (2005). Bref, on se retrouve dans une situation où l'émergence et l'intégration des nouvelles technologies produisent plutôt des comportements inverses aux «lean behavior » recherchés.

Bien que les technologies numériques appliquées au changement de comportement aient un potentiel d'amplification des comportements, de promotion des nouveaux comportements et de maintien d'une dynamique de changement comportemental, leur introduction dans les milieux de travail n'est pas nécessairement fluide. Hormis le problème bien connu de la sécurité des données, au moins deux obstacles peuvent défaire tout leur potentiel à savoir l'acceptabilité sociale et la santé psychologique des personnes exposées à ces technologies. En plus, des voix comme celles PacauxLemoine et de Trentesaux (2019) s'élèvent pour soulever les risques éthiques que posent l'arrivée de 
ces technologies (Pacaux-Lemoine \& Trentesaux, 2019). C'est pourquoi seul le développement de ces technologies ne suffit pas pour impulser et maintenir une nouvelle dynamique comportementale. Elles doivent être accompagnées des stratégies de gestion qui aplanissent tout sentiment négatif visà-vis de ces technologies au moment de leur introduction dans les milieux de travail.

\subsection{Bilan de la revue de littérature}

\subsubsection{Principales conclusions}

De cette revue de littérature, il en ressort que la production épurée est un ensemble de pratiques en gestion industrielle qui vise à minimiser sinon éliminer les 7 types de gaspillage que l'on retrouve dans un système de production. Les pratiques de la production épurée sont celles qui créent de la valeur pour toutes les parties prenantes de l'entreprise que sont les fournisseurs, les clients, les employés, les employeurs et les gouvernements. Les méthodes et outils techniques de la production épurée encore appelée " hard practices » peuvent être structurés en 5 groupes à savoir le Juste-àTemps (JIT), la Total Productive Maintenance (TPM), la Total Quality Management (TQM), le Conctinuous Improvement (CI) et le Human Resource Management (HRM). Quels que soient le secteur d'activité et le contexte, les pratiques de la production épurée ont un impact positif sur la performance opérationnelle, financière et environnementale des entreprises manufacturières. La production épurée est donc une clé indispensable pour l'accroissement de la productivité des entreprises.

Les méthodes et outils techniques de la production épurée peuvent être modernisés avec les technologies de l'industrie 4.0, mais ces technologies toutes seules, ne peuvent pas résoudre l'ensemble des problèmes que l'on retrouve dans une entreprise manufacturière. Pour plus d'efficacité, elles peuvent être combinées aux méthodes et outils techniques de la production épurée avec qui elles ont une relation de complémentarité. Les technologies de l'industrie 4.0 servent de catalyseur aux « hard practices » de la production épurée. Essentiellement, ces technologies aident à la collecte, le traitement et la transmission des données numériques en temps réel ce qui a pour effet de propulser et pérenniser la performance opérationnelle des entreprises. L'industrie 4.0 est un ensemble de 8 technologies régulièrement cité dans la littérature. Celle-ci sont : l'internet des objets (IoT), les systèmes cyberphysiques (CPS), le cloud computing (CC), le big data (BD), les machines autonomes, robots (MA), la réalité augmentée (RA), les systèmes de simulation (SS) et la fabrication additive (FA). Cependant, pour certaines applications de l'industrie 4.0, l'intelligence artificielle (IA), l'intégration horizontale et verticale (IHV), la cyber sécurité (CS) et la communication machine to machine (M2M) sont indispensables.

À côté des « hard practices » de la production épurée, il existe des «soft practices » qui font référence aux relations et comportements humains dont la manifestation au sein des entreprises conditionnement aussi le succès de la production épurée. Certaines dimensions du comportement des équipes de travail sous le nom de "lean behaviors » ont été identifiées comme étant favorables à la production épurée. En plus du niveau de maturité de l'équipe, un comportement humain se manifeste lorsque certains facteurs internes ou externes à la personne sont réunis. Ces facteurs dépendent des approches de la psychologie que sont le behaviorisme, la psychologie cognitive, la psychothérapie systémique et l'économie comportementale. La bonne connaissance de ces facteurs permet de structurer un modèle de changement comportemental ou de culture favorable à la production épurée. Les nouvelles technologies auxquelles appartient l'industrie 4.0 ont le potentiel d'influencer si ce n'est de modifier les comportements humains. Ce potentiel repose sur les 13 principes énoncés par Fogg (2003). Elles peuvent amplifier les comportements déjà existants, promouvoir les nouveaux comportements, et maintenir une dynamique de changement comportemental. 
$\mathrm{Au}$ risque de se tromper, nous constatons après cette revue de littérature que l'utilisation des technologies de l'industrie 4.0 pour la promotion des «lean behaviors » qui sont de la famille des « soft practices » de la production épurée n'a pas encore fait l'objet d'une proposition couplée d'une étude expérimentale. Ce constat est d'ailleurs soutenu par Buer (2018) qui recommande en priorité de poursuivre la recherche dans le domaine en investiguant l'impact de l'industrie 4.0 sur les «soft practices » de la production épurée. Dans la même lancée, Rosin (2020) quant à lui fait remarquer que le lien entre l'industrie 4.0 et la collaboration dans les équipes de travail n'a pas encore fait l'objet d'une étude spécifique. La collaboration est un des comportements favorables de la production épurée. La littérature actuelle renseigne que les technologies de l'industrie 4.0 peuvent effectuer des observations ou des mesures sur les systèmes de production par la collecte et le traitement des données. Si ces mesures sont effectivement effectuées, elles peuvent être exploitées pour mettre en place des stratégies qui facilitent les «lean behaviors » en production épurée. Des travaux de recherche et des développements restent encore à faire pour y parvenir et marque ainsi l'opportunité de recherche qui se dégage après cette revue. Suite à cette opportunité, nous présentons à la section suivante une esquisse de solution qui sera entièrement développée et publiée dans un prochain article.

\section{Esquisse de solution basée sur les objets connectés pour la promotion d'une culture favorable à la production épurée}

La solution proposée dans ce papier consiste à développer un outil technique intégrant les objets connectés qui est capable de détecter en continu les 7 types de gaspillage dans un système manufacturier. Ensuite, combiner cette détection de gaspillage à au moins une approche de changement comportemental afin de susciter auprès des opérationnels de première ligne l'adoption des comportements favorables à la production épurée. Cela aura pour effet de réussir l'opérationnalisation de la production épurée qui ensuite impactera positivement la productivité des entreprises manufacturières. Cette idée est comparable au travail effectué par Finkelstein (2016) dans le domaine de la santé pour susciter l'activité physique chez les personnes et ainsi améliorer leurs états de santé (Finkelstein et al., 2016).

L'outil technique couplé à l'approche de changement comportemental forme un système. Le système de base qui est envisagé dans ce papier est celui que nous appelons le système «lean coach $C N V »$. On se limitera à une présentation sommaire de celui-ci et un exemple d'application permettra de l'illustrer.

\subsection{Présentation succincte du système «lean coach CNV »}

La CNV fait référence à la Communication Non Violente aussi connue sous les appellations communication empathique et communication bienveillante. C'est un processus de communication mis au point par Rosenberg (2018). Il est constitué de 4 étapes : Observation - Sentiment - Besoin Demande (M. B. Rosenberg, 2018). Une des caractéristiques de la CNV est qu'elle permet d'établir une relation d'empathie et authentique avec les autres ce qui engendre la bienveillance.

Pour au moins 2 raisons, nous pensons que ce processus de communication peut être appliqué dans une entreprise manufacturière pour demander avec empathie aux membres des équipes de travail d'ajuster leurs comportements afin qu'ils soient favorables à la production épurée. Premièrement, la communication est essentielle pour la construction d'une bonne équipe de travail (Pentland, 2012). Deuxièmement l'empathie et la fluidité de la communication sont identifiées comme deux facteurs qui influencent la performance d'une équipe (Woolley et al., 2010). En communiquant de manière sincère, authentique et empathique avec ses collègues dans une équipe de travail, ceux-ci sont beaucoup moins perçus comme des adversaires et donc, plus comme des 
collaborateurs. La pratique de la CNV peut alors servir de ferment au sein des équipes de travail pour cultiver les «lean behaviors » indispensables à l'opérationnalisation de la production épurée dans une entreprise.

À la première étape du processus de la CNV, l'observation d'une situation est comparable à un enregistrement effectué par une caméra (Beck, 2005). Le système «lean coach CNV》 que nous proposons consiste à se servir de l'outil technique basé sur les objets connectés comme moyen d'observation. En intégrant les objets connectés pour détecter (observer) les gaspillages, on obtient une description objective et mesurable d'un ensemble de faits de gaspillage qui sont incontestables. Une fois que l'outil technique a effectué l'observation, les 3 autres étapes du processus de la CNV sont appliquées par un facilitateur de la production épurée avec les différentes parties prenantes. Le système «lean coach $C N V$ » se résume à la pratique de la $\mathrm{CNV}$ au sein des équipes de travail par le biais d'un outil technique intégrant les objets connectés comme moyen d'observation. Le schéma de la figure 4.1 montre les éléments constitutifs du système «lean coach $C N V$ ».
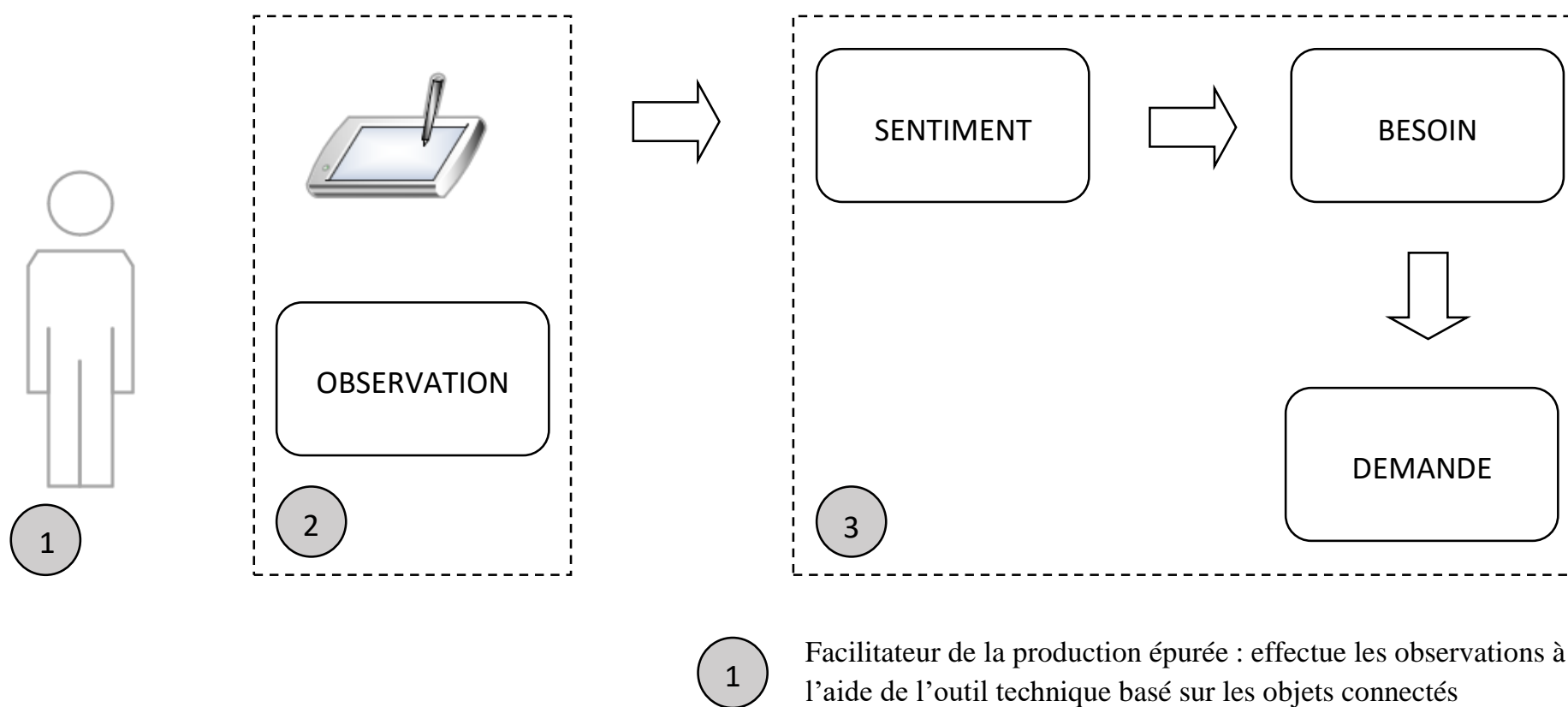

Facilitateur de la production épurée : effectue les observations à l'aide de l'outil technique basé sur les objets connectés

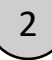

Outil technique : Identifie automatiquement les 7 gaspillages ce qui permet l'observation, première étape du processus $\mathrm{CNV}$

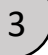

Processus CNV : Après la première étape de la CNV effectuée avec l'outil technique, le facilitateur complète les étapes restantes

Figure 4.1. Schéma illustratif du système «lean coach CNV »

\subsection{Exemple d'application du système «lean coach CNV »}

Cet exemple est une mise en situation qui montre l'application du système "lean coach CNV » afin de susciter l'adoption des comportements favorables à la production épurée dans une équipe de production.

Nous sommes dans le contexte d'une usine qui a la taille d'une PME dont le seul moyen de production est une ligne d'assemblage de plusieurs types de produits. À la sortie de la ligne, les produits finis emballés dans les boites de carton sont empilés sur une palette. Lorsque le nombre 
maximal de boites d'une palette est atteint, un opérateur utilise l'unique chariot élévateur de l'usine pour déplacer la palette et la ranger au magasin des produits finis. Ce jour après avoir produit les 10 premières palettes de son quart de travail sans difficulté, cet opérateur cherche en vain le chariot élévateur dans l'usine pour déplacer la 11ème palette qui vient d'être terminée. Il fait plusieurs vaet-vient dans toutes les directions de l'usine pendant environ 45 minutes. Le magasinier surpris de le voir tourner en rond l'informe après l'avoir demandé ce qu'il cherche que le chariot élévateur se trouve à l'extérieur, car il vient de s'en servir pour réceptionner une livraison. Pendant ces 45 minutes, toute la ligne est en arrêt et les 6 autres opérateurs en attente. En effet, le 7ème opérateur posté à la sortie de la ligne est celui qui empile les boites sur la palette et correspond à celui qui est à la recherche du chariot.

L'outil technique que nous proposons dans cet article intégrera les objets connectés et pourrait géolocaliser les moyens matériels et humains impliqués dans la production. Dans le cas de cette mise en situation, il collectera et enregistrera les données de déplacement de l'opérateur et les traduira en gaspillage de type mouvement inutile au sens de Ohno (1988), Shingo (1989) et Liker (2004). Le facilitateur de la production épurée qui se sert de cet outil technique a le moyen de se rendre compte que le 7ème opérateur a effectué un gaspillage de type mouvement inutile. D'une certaine façon, il fait une observation. Ensuite, il applique les autres étapes de la Communication Non Violente (CNV) afin de demander avec empathie aux opérateurs d'utiliser la technique des $5 \mathrm{~S}$ pour le rangement du matériel de travail. Dans ce cas il s'agit du chariot élévateur. Le 5S est une des techniques de la production épurée qui se rattache au Juste-à-Temps (JIT). La bienveillance, un des «lean behavior » qui serait cultivé par le système «lean coach $C N V$ » motiverait les opérateurs à appliquer le $5 \mathrm{~S}$ comme pratique de travail. Dans cette mise en situation, 45 minutes seraient convertibles en gain de productivité et personne ne se sentirait blessé.

L'application du système «lean coach $C N V$ » dans cet exemple n'a été possible parce que les objets connectés intégrés ont été utilisés pour observer, enregistrer et interpréter le gaspillage de type mouvement inutile au sens de Ohno (1988), Shingo (1989) et Liker (2004). C'est en cela que les objets connectés ont été utiles pour promouvoir les comportements favorables à la production épurée. L'outil technique que nous proposons pourra faire la même chose pour les autres types de gaspillage et le développement complet du système pourrait inciter à plus de comportements favorables à la production épurée. Cette production épurée aura à son tour un impact sur la productivité des entreprises.

\section{Conclusion}

L'objectif de ce papier a été de montrer l'importance des objets connectés pour la promotion des comportements favorables à la production épurée. En soi, la production épurée ou production amincie est un résultat qu'il faut entretenir une fois qu'il est acquis. Pour cela il faut traiter sans relâche les gaspillages avec les méthodes et outils qui structurent une stratégie «lean ». La proposition contenue dans ce papier consiste à se servir d'un outil technique pour détecter en continu les 7 types de gaspillage dans un système manufacturier et de combiner cette détection de gaspillage aux approches de changement comportemental pour semer une culture de la production épurée. Cette culture passe par la Communication Non Violente (CNV) qui nourrit la bienveillance et la collaboration indispensables à la mise en œuvre des méthodes et outils techniques de la production épurée. Nous pensons que la contribution de cette proposition se situe à 2 niveaux. 
Premièrement l'intégration des objets connectés pour détecter en continu les 7 types de gaspillage dans un système manufacturier et déclencher les actions d'amélioration. Deuxièmement l'application de la CNV comme facteur d'adoption des comportements favorables à la production épurée. Cette contribution dans le domaine de la gestion industrielle aura de façon directe un impact sur la productivité des entreprises et de façon indirecte sur l'incitation des entreprises au passage à l'industrie 4.0. Sur le plan académique, l'impact sera sur l'actualisation de l'enseignement de la production épurée.

\section{Bibliographie}

Ahuja, I., \& Kumar, P. (2009). A case study of total productive maintenance implementation at precision tube mills. Journal of Quality in Maintenance Engineering, 15, 241-258. https://doi.org/10.1108/13552510910983198

Almquist, E., Senior, J., \& Bloch, N. (2016, septembre). The elements of value. Harvard Business Review. https://hbr.org/2016/09/the-elements-of-value

Amjad, M. S., Rafique, M. Z., \& Khan, M. A. (2021). Leveraging Optimized and Cleaner Production through Industry 4.0. Sustainable Production and Consumption, 26, 859-871. https://doi.org/10.1016/j.spc.2021.01.001

Amrani, A., \& Ducq, Y. (2020). Lean practices implementation in aerospace based on sector characteristics: Methodology and case study. Production Planning \& Control, 1-23. https://doi.org/10.1080/09537287.2019.1706197

Anand, G., Ward, P. T., Tatikonda, M. V., \& Schilling, D. A. (2009). Dynamic capabilities through continuous improvement infrastructure. Journal of Operations Management, 27(6), 444-461. https://doi.org/10.1016/j.jom.2009.02.002

Armstrong, M., \& Taylor, S. (2014). Armstrong's Handbook of human resource management practice (13e éd.). Kogan Page Ltd.

ASPAM. (2021). Santé psychologique—Vue globale. https://www.apsam.com/theme/sante-psychologique/santepsychologique-vue-globale

Beck, S. (2005). Developing nonviolent communication : An integral approach. University of Victoria.

Belekoukias, I., Garza-Reyes, J. A., \& Kumar, V. (2014). The impact of lean methods and tools on the operational performance of manufacturing organisations. International Journal of Production Research, 52(18), 5346-5366. https://doi.org/10.1080/00207543.2014.903348

Benghozi, P.-J., Bureau, S., \& Massit-Folléa, F. (2009). L'Internet des objets quels enjeux pour l'Europe? Éditions de la Maison des sciences de l'homme.

Bevilacqua, M., Ciarapica, F. E., \& De Sanctis, I. (2017). Lean practices implementation and their relationships with operational responsiveness and company performance: An Italian study. International Journal of Production Research, 55(3), 769-794. https://doi.org/10.1080/00207543.2016.1211346

Bidet-Mayer, T. (2016). L'industrie du futur : Une compétition mondiale. Presses des Mines.

Borcsa, M., \& Stratton, P. (2016). Origins and originality in family therapy and systemic practice. Springer.

Brown, P. M., Cameron, L. D., Wilkinson, M., \& Taylor, D. (2020). Economic and Behavioral Economic Approaches to Behavior Change. In K. Hamilton, L. D. Cameron, M. S. Hagger, N. Hankonen, \& T. Lintunen (Éds.), The Handbook of Behavior Change (p. 617-631). Cambridge University Press; Cambridge Core. https://doi.org/10.1017/9781108677318.042

Buer, S.-V., Semini, M., Strandhagen, J. O., \& Sgarbossa, F. (2021). The complementary effect of lean manufacturing and digitalisation on operational performance. International Journal of Production Research, 59, 1-17. https://doi.org/10.1080/00207543.2020.1790684

Buer, S.-V., Strandhagen, J. O., \& Chan, F. T. S. (2018). The link between Industry 4.0 and lean manufacturing: Mapping current research and establishing a research agenda. International Journal of Production Research, 56(8), 2924-2940. https://doi.org/10.1080/00207543.2018.1442945

Cascio, W., \& Montealegre, R. (2016). How Technology Is Changing Work and Organizations. Annual Review of Organizational Psychology and Organizational Behavior, 3, 349-375. https://doi.org/10.1146/annurev-orgpsych041015-062352 
CEFRIO. (2016). Prendre part à la révolution manufacturière? Du rattrapage technologique à l'Industrie 4.0 chez les PME. CEFRIO.

Cherry, K. (2019). Cognitive psychology: The science of how we think. Verywellmind. https://www.verywellmind.com/cognitive-psychology-4157181

Ciano, M., Dallasega, P., Orzes, G., \& Rossi, T. (2021). One-to-one relationships between Industry 4.0 technologies and Lean Production techniques: A multiple case study. International Journal of Production Research, 1, 25. https://doi.org/10.1080/00207543.2020.1821119

Čiarnienè, R., \& Vienažindienè, M. (2015). An Empirical Study of Lean Concept Manifestation. 11th International Strategic Management Conference, 207, 225-233. https://doi.org/10.1016/j.sbspro.2015.10.091

Cohen, D. (2015). HR past, present and future : A call for consistent practices and a focus on competencies. Human Resource Management Review, 25. https://doi.org/10.1016/j.hrmr.2015.01.006

Concordia University. (2020). How to write a literature review. https://library.concordia.ca/help/writing/literaturereview.php

Côté, P. (2018). La contribution de l'économie comportementale aux questions environnementale: En quête d'un nouveau paradigme [Maîtrise]. Université de Sherbrooke.

Cua, K. O., McKone, K. E., \& Schroeder, R. G. (2001). Relationships between implementation of TQM, JIT, and TPM and manufacturing performance. Journal of Operations Management, 19(6), 675-694. https://doi.org/10.1016/S0272-6963(01)00066-3

Darlington, J., Francis, M., Found, P., \& Thomas, A. (2015). Targeting lean process improvement projects for maximum financial impact. Production Planning and Control, 27. https://doi.org/10.1080/09537287.2015.1082665

Davies, R., Coole, T., \& Smith, A. (2017). Review of Socio-technical Considerations to Ensure Successful Implementation of Industry 4.0. Procedia Manufacturing, 11, 1288-1295. https://doi.org/10.1016/j.promfg.2017.07.256

Dombrowski, U., \& Richter, T. (2018). The Lean Production System 4.0 Framework - Enhancing Lean Methods by Industrie 4.0: IFIP WG 5.7 International Conference, APMS 2018, Seoul, Korea, August 26-30, 2018, Proceedings, Part II (p. 410-416). https://doi.org/10.1007/978-3-319-99707-0_51

Dombrowski, U., Richter, T., \& Krenkel, P. (2017). Interdependencies of Industrie 4.0 \& Lean Production Systems : A Use Cases Analysis. Procedia Manufacturing, 11, 1061-1068. https://doi.org/10.1016/j.promfg.2017.07.217

Dora, M., Kumar, M., Goubergen, D. V., Molnar, A., \& Gellynck, X. (2013). Operational performance and critical success factors of lean manufacturing in European food processing SMEs. Trends in Food Science \& Technology, 31, 156-164. http://dx.doi.org/10.1016/j.tifs.2013.03.002

Drath, R., \& Horch, A. (2014). Industrie 4.0 : Hit or Hype? [Industry Forum]. Industrial Electronics Magazine, IEEE, 8, 56-58. https://doi.org/10.1109/MIE.2014.2312079

Dubey, R., \& Singh, T. (2015). Understanding complex relationship among JIT, lean behaviour, TQM and their antecedents using interpretive structural modelling and fuzzy MICMAC analysis. The TQM Journal, 27, 42-62. https://doi.org/10.1108/TQM-09-2013-0108

El Hakim, A. (2018). Internet of Things (IoT) System Architecture and Technologies, White Paper. https://doi.org/10.13140/RG.2.2.17046.19521

Emiliani, M. L. (1998). Lean behaviors. Management Decision, 36, 615-631. https://doi.org/10.1108/00251749810239504

Finkelstein, E. A., Haaland, B. A., Bilger, M., Sahasranaman, A., Sloan, R. A., Nang, E. E. K., \& Evenson, K. R. (2016). Effectiveness of activity trackers with and without incentives to increase physical activity (TRIPPA): A randomised controlled trial. The Lancet Diabetes \& Endocrinology, 4(12), 983-995. https://doi.org/10.1016/S22138587(16)30284-4

Fiore, S. M., Salas, E., \& Cannon-Bowers, J. A. (2001). Group dynamics and shared mental model development. In How people evaluate others in organizations. (p. 309-336). Lawrence Erlbaum Associates Publishers.

Fogg, B. J. (2003). Persuasive technology: Using computers to change what we think and do. Morgan Kaufmann Publishers.

Fullerton, R. R., Kennedy, F. A., \& Widener, S. K. (2014). Lean manufacturing and firm performance: The incremental contribution of lean management accounting practices. Journal of Operations Management, 32, 414-428. https://doi.org/10.1016/j.jom.2014.09.002 
Furlan, A., Vinelli, A., \& Pont, G. (2011). Complementarity and lean manufacturing bundles : An empirical analysis. International Journal of Operations \& Production Management, 31, 835-850. https://doi.org/10.1108/01443571111153067

Gallup. (2021). State of the global workplace. Gallup Press. https://www.gallup.com/workplace/349484/state-of-theglobal-workplace.aspx

Garza-Reyes, J. A., Kumar, V., Chaikittisilp, S., \& Tan, K. H. (2018). The effect of lean methods and tools on the environmental performance of manufacturing organisations. International Journal of Production Economics, 200, 170-180. https://doi.org/10.1016/j.ijpe.2018.03.030

Georgia State University. (2017). Approach of psychology. http://sites.gsu.edu/zbhatt1/

Gerbert, P., Lorenz, M., Rüßmann, M., Waldner, M., Justus, J., Engel, P., \& Harnisch, M. (2015). Industry 4.0 : The Future of Productivity and Growth in Manufacturing Industries. Boston Consulting Group.

Gergen, K. J. (1982). Toward a transformation in social knowledge. Verlag.

Gibson, J. (1979). The Ecological Approach to Visual Perception. Houghton-Mifflin.

Gino, F. (2017). The Rise of Behavioral Economics and Its Influence on Organizations. https://hbr.org/2017/10/therise-of-behavioral-economics-and-its-influence-on-organizations

Glover, W. J., Farris, J. A., \& Van Aken, E. M. (2015). The relationship between continuous improvement and rapid improvement sustainability. International Journal of Production Research, 53(13), 4068-4086. https://doi.org/10.1080/00207543.2014.991841

Gouvernement du Québec. (2020, mai). Production à valeur ajoutée (PVA). Guides et outils. https://www.economie.gouv.qc.ca/bibliotheques/outils/gestion-dune-entreprise/production/

Graham, G. (2019). Behaviorism. Stanford Encyclopedia of Philosophy. https://plato.stanford.edu/search/searcher.py?query=Behaviorism

Gupta, B. B., \& Quamara, M. (2018). An overview of Internet of Things (IoT) : Architectural aspects, challenges, and protocols. Concurrency and Computation: Practice and Experience. https://doi.org/10.1002/cpe.4946

Hayes, A. S. (2020). The Behavioral Economics of Pierre Bourdieu. Sociological Theory, 38(1), 16-35. https://doi.org/10.1177/0735275120902170

Hodge, G. L., Goforth Ross, K., Joines, J. A., \& Thoney, K. (2011). Adapting lean manufacturing principles to the textile industry. Production Planning \& Control, 22(3), 237-247. https://doi.org/10.1080/09537287.2010.498577

Hofer, C., Eroglu, C., \& Rossiter Hofer, A. (2012). The effect of lean production on financial performance: The mediating role of inventory leanness. Int. J. Production Economics, 138, 242-253. https://doi.org/10.1016/j.ijpe.2012.03.025

Hu Qing, Mason Robert, Williams Sharon J., \& Found Pauline. (2015). Lean implementation within SMEs: A literature review. Journal of Manufacturing Technology Management, 26(7), 980-1012. https://doi.org/10.1108/JMTM-02-2014-0013

INSEE. (2020, mai). Valeur ajoutée. Institut national de la statistique et des études économiques. https://www.insee.fr/fr/metadonnees/definition/c1950

Jahchan, P. (2016). What is productivity, and how do you measure it? World Economic Forum. https://www.weforum.org/agenda/2016/07/what-is-productivity-and-how-do-you-measure-it/

Jane, M., Foster, J., Hagger, M., \& Pal, S. (2015). Using new technologies to promote weight management: A randomised controlled trial study protocol. BMC Public Health, 15(1), 509. https://doi.org/10.1186/s12889-015$1849-4$

Johnson, S. (2013). Recognising the true potential of technology to change behaviour. Behavioural insights. https://www.theguardian.com/sustainable-business/behavioural-insights

Joinson, A. N., \& Piwek, L. (2013). Technology and Behaviour Change, for Good and Evil. University of the West of England (UWE).

Kahneman, D. (2011). Thinking, fast and slow. Farrar, Straus and Giroux.

Kamble, S., Gunasekaran, A., \& Dhone, N. C. (2020). Industry 4.0 and lean manufacturing practices for sustainable organisational performance in Indian manufacturing companies. International Journal of Production Research, 58(5), 1319-1337. https://doi.org/10.1080/00207543.2019.1630772 
Karikalan, R., Sreeharan, B., Akilan, S., \& Rallish, R. K. (2019). Productivity improvement using Lean concept in automotive welding fixture manufacturing industry. International Journal of Innovative Technology and Exploring Engineering, 8(9S2), 427-431. https://doi.org/10.35940/ijitee.I1091.0789S219

Kashav, S., Centobelli, P., Cerchione, R., \& Singh, R. (2019). The impact of leanness and innovativeness on environmental and financial performance: Insights from Indian SMEs. International Journal of Production Economics, 212. https://doi.org/10.1016/j.ijpe.2019.02.011

Kaynak, H. (2003). The Relationship Between Total Quality Management Practices and Their Effects on Firm Performance. Journal of Operations Management, 21. https://doi.org/10.1016/S0272-6963(03)00004-4

Kinley, N., \& Ben-Hur, S. (2015). Changing employee behavior. PALGRAVE MACMILLAN.

Kolberg, D., Knobloch, J., \& Zühlke, D. (2017). Towards a lean automation interface for workstations. International Journal of Production Research, 55(10), 2845-2856. https://doi.org/10.1080/00207543.2016.1223384

Konecny, P. A., \& Thun, J.-H. (2011). Do it separately or simultaneously-An empirical analysis of a conjoint implementation of TQM and TPM on plant performance. Towards High Performance Manufacturing, 133(2), 496-507. https://doi.org/10.1016/j.ijpe.2010.12.009

Kummer, T.-F., Recker, J., \& Bick, M. (2017). Technology-induced anxiety : Manifestations, cultural influences, and its effect on the adoption of sensor-based technology in German and Australian hospitals. Information \& Management, 54(1), 73-89. https://doi.org/10.1016/j.im.2016.04.002

Lake, M. (2018). How new technology is driving real-time behavior change in organizational transformations. IT Talent Revolution. https://www.cio.com/blog/it-talent-revolution/

Liker, J. (2004). The Toyota way: 14 management principles from the world's greatest manufacturer. McGraw-Hill Education.

Lilly, J., \& Durr, D. (2012). Technology changes at work and employee reactions : The role of leader behavior. Human Systems Management, 31, 193-201. https://doi.org/10.3233/HSM-2012-0769

Lima, M. J. do R. F., Todaro, M. C., \& Rocha, M. dos S. (2018). A methodological approach for kaizen events in assembly lines. Journal of Lean Systems, 3(1), 46-65.

Lock, A., \& Strong, T. (2010). Social Constructionism: Sources and Stirrings in Theory and Practice. In Social Constructionism : Sources and Stirrings in Theory and Practice. https://doi.org/10.1017/CBO9780511815454

Locke, E. A. (2009). Handbook Of Principles Of Organizational Behavior : Indispensable Knowledge For EvidenceBased Management. John Wiley \& Sons.

Lyonnet, B. (2010). Amélioration de la performance industrielle: Vers un système de production Lean adapté aux entreprises du pôle de compétitivité Arve Industries Haute-Savoie Mont-Blanc [Thèse, Université de Savoie]. Archives-ouvertes. https://tel.archives-ouvertes.fr/tel-00655808/

Lyonnet, B., Pillet, M., \& Pralus, M. (2010). Lean manufacturing in the screw cutting sector : Assessment of maturity level. International Journal of Rapid Manufacturing, 1(3). https://doi.org/10.1504/IJRAPIDM.2010.034249

M. Hermann, T. Pentek, \& B. Otto. (2016). Design Principles for Industrie 4.0 Scenarios. 2016 49th Hawaii International Conference on System Sciences (HICSS), 3928-3937. https://doi.org/10.1109/HICSS.2016.488

Machi, L., \& McEvoy, B. (2012). The literature review six steps to success (2nd edition). Corwin.

Magd Hesham \& Curry Adrienne. (2003). ISO 9000 and TQM: are they complementary or contradictory to each other? The TQM Magazine, 15(4), 244-256. https://doi.org/10.1108/09544780310486155

Maginnis, M., Hapuwatte, B., \& Keown, D. (2020). The Integration of True Lean and Industry 4.0 to Sustain a Culture of Continuous Improvement (p. 336-345). https://doi.org/10.1007/978-3-030-42250-9_32

Manoj Kumar, N., \& Mallick, P. K. (2018). The Internet of Things : Insights into the building blocks, component interactions, and architecture layers. Procedia Computer Science, 132, 109-117. https://doi.org/10.1016/j.procs.2018.05.170

Massachusetts Institute of Technology. (2020). MIT AUTO-ID LABORATORY. https://autoid.mit.edu/

Mathur, A., Mittal, M. L., \& Dangayach, G. S. (2012). Improving productivity in Indian SMEs. Production Planning \& Control, 23(10-11), 754-768. https://doi.org/10.1080/09537287.2011.642150

Mayr, A., Weigelt, M., Kühl, A., Grimm, S., Erll, A., Potzel, M., \& Franke, J. (2018). Lean 4.0-A conceptual conjunction of lean management and Industry 4.0. https://doi.org/10.1016/j.procir.2018.03.292 
McLeod, S. (2017). Behaviorist approach. https://www.simplypsychology.org/behaviorism.html

McNamee, S., \& Gergen, K. J. (1992). Therapy as social construction. Sage.

Meuter, M. L., Bitner, M. J., Ostrom, A. L., \& Brown, S. W. (2005). Choosing among Alternative Service Delivery Modes: An Investigation of Customer Trial of Self-Service Technologies. Journal of Marketing, 69(2), 61-83. https://doi.org/10.1509/jmkg.69.2.61.60759

Michaud, F. (2014). Utilisation des technologies persuasives dans le domaine du transport: Bonnes et mauvaises pratiques web pour favoriser le covoiturage [Université de Montréal]. Polypublie. https://publications.polymtl.ca/1618/

Nawanir, G., Teong, L. K., \& Othman, S. N. (2013). Impact of lean practices on operations performance and business performance: Some evidence from Indonesian manufacturing companies. Journal of Manufacturing Technology Management, 24(7), 1019-1050. https://doi.org/10.1108/JMTM-03-2012-0027

Negrão, L. L. L., Godinho Filho, M., \& Marodin, G. (2017). Lean practices and their effect on performance: A literature review. Production Planning \& Control, 28(1), 33-56. https://doi.org/10.1080/09537287.2016.1231853

OCDE. (2021). OECD Compendium of Productivity Indicators. Éditions OCDE. https://doi.org/10.1787/f25cdb25-en.

Ohno, T. (1988). Toyota Production System : Beyond large-scale production. CRC Press, Taylor \& Francis Group.

Pacaux-Lemoine, M.-P., \& Trentesaux, D. (2019). Ethical risks of human-machine symbiosis in industry 4.0 : Insights from the human-machine cooperation approach. 14th IFAC Symposium on Analysis, Design, and Evaluation of Human Machine Systems HMS 2019, 52(19), 19-24. https://doi.org/10.1016/j.ifacol.2019.12.077

Pagliosa, M., Tortorella, G., \& Ferreira, J. (2019). Industry 4.0 and Lean Manufacturing: A Systematic Literature Review and Future Research Directions. Journal of Manufacturing Technology Management, 31. https://doi.org/10.1108/JMTM-12-2018-0446

Pamela, D. (2018). Can technology drive organizational culture? Deep Dive. https://www.hrdive.com/

Panwar, A., Jain, R., Rathore, A. P. S., Nepal, B., \& Lyons, A. (2018). The impact of lean practices on operational performance - an empirical investigation of Indian process industries. Production Planning \& Control, 29(2), 158-169. https://doi.org/10.1080/09537287.2017.1397788

Pavnaskar, S. J., Gershenson, J. K., \& Jambekar, A. B. (2003). Classification scheme for lean manufacturing tools. International Journal of Production Research, 41(13), 3075-3090. https://doi.org/10.1080/0020754021000049817

Pea, R. (1985). Beyond Amplification: Using the Computer to Reorganize Mental Functioning. Educational Psychologist, 20. https://doi.org/10.1207/s15326985ep2004_2

Pearce, A., Pons, D., \& Neitzert, T. (2018). Implementing lean-Outcomes from SME case studies. Operations Research Perspectives, 5, 94-104. https://doi.org/10.1016/j.orp.2018.02.002

Pentland, A. S. (2012). The new science of building great teams. Harvard Business Review.

Pfaffinger, K. F., Reif, J. A. M., Spieß, E., \& Berger, R. (2020). Anxiety in a digitalised work environment. Gruppe. Interaktion. Organisation. Zeitschrift für Angewandte Organisationspsychologie (GIO), 51(1), 25-35. https://doi.org/10.1007/s11612-020-00502-4

Productive Edge Team. (2015). Developing Effective Behavioral Change Technology. Productive/Edge. https://www.productiveedge.com/2015/04/09/developing-effective-behavioral-change-technology/

Rosenberg, M. B. (2018). La communication non violente au quotidien. Jouvence.

Rosenberg, M. J. (1960). Attitude organization and change; an analysis of consistency among attitude components, by Milton J. Rosenberg [and others]. Yale University Press; /z-wcorg/.

Rosin, F., Forget, P., Lamouri, S., \& Pellerin, R. (2020). Impacts of Industry 4.0 technologies on Lean principles. International Journal of Production Research, 58(6), 1644-1661. https://doi.org/10.1080/00207543.2019.1672902

Rossini, M., Audino, F., Costa, F., Cifone, F., Kundu, K., \& Portioli-Staudacher, A. (2019). Extending lean frontiers : A kaizen case study in an Italian MTO manufacturing company. The International Journal of Advanced Manufacturing Technology, 104. https://doi.org/10.1007/s00170-019-03990-x

Rossini, M., Costa, F., Tortorella, G. L., \& Portioli-Staudacher, A. (2019). The interrelation between Industry 4.0 and lean production: An empirical study on European manufacturers. The International Journal of Advanced Manufacturing Technology, 102(9), 3963-3976. https://doi.org/10.1007/s00170-019-03441-7 
Rousseau, V., Aubé, C., \& Savoie, A. (2006a). Le fonctionnement interne des équipes de travail : Conception et mesure. / Internal functioning of work teams : Conception and measurement. Canadian Journal of Behavioural Science/Revue canadienne des sciences du comportement, 38, 120-135. https://doi.org/10.1037/cjbs2006002

Rousseau, V., Aubé, C., \& Savoie, A. (2006b). Teamwork Behaviors A Review and an Integration of Frameworks. Small Group Research, 37, 540-570. https://doi.org/10.1177/1046496406293125

Rüttimann, B., \& Stöckli, M. (2016). Lean and Industry 4.0-Twins, Partners, or Contenders ? A Due Clarification Regarding the Supposed Clash of Two Production Systems. Journal of Service Science and Management, 09, 485-500. https://doi.org/10.4236/jssm.2016.96051

Saito, A., Kozo, S., \& Cho, F. (2012). Seeds of collaboration: Seeking the essence of the Toyota Production System, an appreciation of Mr. Fujio Cho, master teacher. Monterey, Ky. : Larkspur Press.

Sanders, A., Subramanian, K., Redlich, T., \& Wulfsberg, J. (2017). Industry 4.0 and Lean Management - Synergy or Contradiction? (p. 349). https://doi.org/10.1007/978-3-319-66926-7_39

Schermerhorn, J. R., Hunt, J. G., Osborn, R. N., \& Claire de Billy. (2014). Comportement humain et organisation. ERPI Sciences administraties.

Schoemaker, E. (2014). The Mobile Web : Amplifying, but Not Creating, Changemakers. Innovations: Technology, Governance, Globalization, 9, 75-85. https://doi.org/10.1162/inov_a_00218

Secchi, R., \& Camuffo, A. (2019). Lean implementation failures: The role of organizational ambidexterity. International Journal of Production Economics, 210, 145-154.

Serpanos, D., \& Wolf, M. (2018). Industrial Internet of Things. In: Internet-of-Things (IoT) Systems. Springer. https://doi.org/10.1007/978-3-319-69715-4_5

Sethi, P., \& Sarangi, S. (2017). Internet of Things : Architectures, Protocols, and Applications. Journal of Electrical and Computer Engineering, 2017, 1-25. https://doi.org/10.1155/2017/9324035

Shah, R., \& Ward, P. T. (2003). Lean manufacturing: Context, practice bundles, and performance. Journal of Operations Management, 21(2), 129-149. https://doi.org/10.1016/S0272-6963(02)00108-0

Shahin, M., Chen, F. F., Bouzary, H., \& Krishnaiyer, K. (2020). Integration of Lean practices and Industry 4.0 technologies: Smart manufacturing for next-generation enterprises. The International Journal of Advanced Manufacturing Technology, 107(5), 2927-2936. https://doi.org/10.1007/s00170-020-05124-0

Shingo, S. (1989). A study of the Toyota Production System from an industrial engineering Viewpoint. Productivity Press.

Singh, H., \& Singh, B. (2014). Total Quality Management: Today's Business Excellence Strategy. International Letters of Social and Humanistic Sciences, 32, 188-196. https://doi.org/10.18052/www.scipress.com/ILSHS.32.188

Singler, É. (2015). Green Nudge : Réussir à changer les comportements pour sauver la planète. Pearsons.

Slack, N., Brandon-Jones, A., \& Johnston, R. (2013). Operations management. Pearsons.

Soash, R. L. (1999). Media equation: How people treat computers, television, and new media like real people and places. Collection Management, 24(3-4), 310-311. https://doi.org/10.1300/J105v24n03_14

Solow, R. (1987). We’’d Better Watch Out. New York Times Book Review, 36.

Spear, S., \& Bowen, H. K. (1999). Decoding the DNA of the Toyota production system. Harvard Business Review.

Staufen AG. (2015). China - Industry 4.0 Index 2015. Staufen.

Stevenson, W. J., \& Benedetti, C. (2011). La gestion des opérations, produits et services. Chenelière, McGraw-Hill.

STIQ. (2020). Baromètre industriel québécois (11e édition). https://www.stiq.com/barometre-industriel-quebecois/

Taj, S., \& Morosan, C. (2011). The impact of lean operations on the Chinese manufacturing performance. Journal of Manufacturing Technology Management, 22(2), 223-240. https://doi.org/10.1108/17410381111102234

Thaler, R., \& Sunstein, C. (2009). NUDGE: Improving Decisions About Health, Wealth, and Happiness. In Nudge: Improving Decisions about Health, Wealth, and Happiness (Vol. 47).

Thürer, M., Tomašević, I., \& Stevenson, M. (2017). On the meaning of 'Waste' : Review and definition. Production Planning \& Control, 28(3), 244-255. https://doi.org/10.1080/09537287.2016.1264640 
Tortorella, G. L., \& Fettermann, D. (2018). Implementation of Industry 4.0 and lean production in Brazilian manufacturing companies. International Journal of Production Research, 56(8), 2975-2987. https://doi.org/10.1080/00207543.2017.1391420

Toyama, K. (2015). Geek Heresy: Rescuing Social Change from the Cult of Technology. PublicAffairs.

Tuckman, B. W. (1965). Developmental sequence in small groups. Psychological Bulletin, 63.

Valentin, J., Coudret, F., Gouardères, E., \& Lefer, W. (2012). Modélisation du comportement humain pour la simulation d'évacuation de bâtiment en feu. 5.

Van Acker, B., Conradie, P., Vlerick, P., \& Saldien, J. (2019). Employee Acceptability of Wearable Mental Workload Monitoring in Industry 4.0: A Pilot Study on Motivational and Contextual Framing. Proceedings of the Design Society: International Conference on Engineering Design, 1, 2101-2110. https://doi.org/10.1017/dsi.2019.216

Wagner, T., Herrmann, C., \& Thiede, S. (2017). Industry 4.0 Impacts on Lean Production Systems. Procedia CIRP, 63, 125-131. https://doi.org/10.1016/j.procir.2017.02.041

Wertheimer, M., \& Puente, A. E. (2020). A brief history of psychology. Routledge.

Womack, J., \& Jones, D. (2003). Lean Thinking: Banish waste and create wealth in your corporation. Free Press.

Womack, J., Jones, D., \& Roos, D. (1990). The machine that changed the world. Harper Collins.

Woolley, A., Chabris, C., Pentland, A., Hashmi, N., \& Malone, T. (2010). Evidence of a Collective Intelligence Factor in the Performance of Human Groups. Science (New York, N.Y.), 330, 686-688. https://doi.org/10.1126/science.1193147

X. Liu, K. H. Lam, K. Zhu, C. Zheng, X. Li, Y. Du, C. Liu, \& P. W. T. Pong. (2019). Overview of Spintronic Sensors With Internet of Things for Smart Living. IEEE Transactions on Magnetics, 55(11), 1-22. https://doi.org/10.1109/TMAG.2019.2927457

Yang, M. G. (Mark), Hong, P., \& Modi, S. B. (2011). Impact of lean manufacturing and environmental management on business performance : An empirical study of manufacturing firms. Int. J. Production Economics, 129, 251-261.

Yash, D. (2016). Development of integrated model for improving productivity through lean manufacturing practices [Institute of Engineering \& Technology]. Shodhganga. http://hdl.handle.net/10603/235244

Zajkowska, E. L. (2013). Contribution à l'implantation de la méthode Lean Six Sigma dans les Petites et Moyennes Entreprises pour l'amélioration des processus [École centrale de Lille]. Archives-ouvertes. https://tel.archivesouvertes.fr/tel-00795533/document 